\title{
First-principles calculation method for electron transport based on the grid Lippmann-Schwinger equation
}

\author{
Yoshiyuki Egami* \\ Faculty of Engineering, Hokkaido University, Sapporo, Hokkaido 060-8628, Japan \\ Shigeru Iwase \\ Graduate School of Engineering, Osaka University, Suita, Osaka 565-0871, Japan \\ Shigeru Tsukamoto \\ Peter Grünberg Institut \& Institute for Advanced Simulation, Forschungszentrum Jülich and JARA, D-52425 Jülich, Germany \\ Tomoya Ono \\ Center for Computational Sciences, University of Tsukuba, Tsukuba, Ibaraki 305-8577, Japan \\ and JST-PRESTO, Kawaguchi, Saitama 332-0012, Japan \\ Kikuji Hirose \\ Graduate School of Engineering, Osaka University, Suita, Osaka 565-0871, Japan
}

(Received 14 April 2015; published 1 September 2015)

\begin{abstract}
We develop a first-principles electron-transport simulator based on the Lippmann-Schwinger (LS) equation within the framework of the real-space finite-difference scheme. In our fully real-space-based LS (grid LS) method, the ratio expression technique for the scattering wave functions and the Green's function elements of the reference system is employed to avoid numerical collapse. Furthermore, we present analytical expressions and/or prominent calculation procedures for the retarded Green's function, which are utilized in the grid LS approach. In order to demonstrate the performance of the grid LS method, we simulate the electron-transport properties of the semiconductor-oxide interfaces sandwiched between semi-infinite jellium electrodes. The results confirm that the leakage current through the $(001) \mathrm{Si}_{-} \mathrm{SiO}_{2}$ model becomes much larger when the dangling-bond state is induced by a defect in the oxygen layer, while that through the $(001) \mathrm{Ge}-\mathrm{GeO}_{2}$ model is insensitive to the dangling bond state.
\end{abstract}

DOI: 10.1103/PhysRevE.92.033301

PACS number(s): 02.70.-c, 73.63.-b, 73.40.-c, 71.15.Mb

\section{INTRODUCTION}

Electron-transport calculations are important tools to investigate and develop materials for new electronic devices. Recently, to obtain more practical knowledge on the electrontransport properties of nanoscale structures, long-range and large-scale transport simulations have attracted much interest. However, such simulations are a very hard task since huge computational costs, growing with the system size, are required. Therefore, it is important to develop an efficient electron-transport simulator.

The Lippmann-Schwinger (LS) equation method proposed by Lang et al. [1-3] is a popular method, which enables us to obtain the scattering wave functions of nanoscale structures sandwiched between electrodes by solving the integral equation of the second-kind Fredholm equation. When the reference system consists of only bare left and right electrodes with an empty transition region, scattering wave functions can be efficiently evaluated for a variety of structures of nanoscale junctions set up in the transition region by using the same reference Green's function of the bare electrode system, where computation of the reference Green's function has to be performed only once. Moreover, for a similar

\footnotetext{
*y_egami@eng.hokudai.ac.jp
}

reason, the LS equation is utilized in the implementation of self-consistent calculations for the convergence of electronic states in infinitely open systems [4-8]. In the conventional LS equation method, scattering wave functions are expressed in the Laue representation; that is, the LS equation is solved by using a two-dimensional plane-wave expansion in the directions parallel to the electrode surface (lateral directions) and a real-space discretization of the coordinate in the direction perpendicular to that (longitudinal direction). In the LS equation method, however, one may frequently encounter a numerical difficulty such that a part of the Green's function expressed in a variable-separable form drastically varies due to the appearance of evanescent waves exponentially growing and decaying in the longitudinal direction. To overcome this issue, in the previous study [8], we proposed the procedure of the ratio expression for the Green's function matrix elements in the Laue representation as a remedy for avoiding numerical collapse.

So far, we have developed several simulators to elucidate the electronic properties of nanostructures based on the realspace finite-difference (RSFD) approach [8-18], in which the system is divided by equally spaced grid points, within the framework of the density functional theory $[19,20]$. For electron-transport simulations, the RSFD method has several advantages compared with the method of the Laue representation from fundamental and practical points of view. 
First, the finite differentiation for the kinetic-energy operator is treated on equal footing in all three directions. This avoids numerical errors due to artificial anisotropy between the lateral and the longitudinal directions at any grid spacing. Second, the computational accuracy can be improved by employing a higher order finite-difference formula. Third, in lateral directions, isolated boundary conditions are available as well as periodic ones, which enables us to treat electrodes as leads. Furthermore, the algorithm of the RSFD method is suitable for massively parallel computing [21].

In this paper, we present the fully real-space-based LS method and the ratio expression technique for the Green's function of the reference system within the RSFD approach. This is referred to as the grid LS method. To demonstrate the performance of the grid LS method, we use it to investigate the electron-transport properties of the (001) $\mathrm{Si}_{-} \mathrm{SiO}_{2}$ and $(001) \mathrm{Ge}-$ $\mathrm{GeO}_{2}$ models connected to semi-infinite jellium electrodes. We also estimate how the dangling bond (DB) caused by an oxygen vacancy contributes to leakage currents across the interface between the semiconductor and the oxide. The results indicate that the leakage current attributed to the $\mathrm{DB}$ state in the $\mathrm{Si}-\mathrm{SiO}_{2}$ model is much larger than that in the $\mathrm{Ge}-\mathrm{GeO}_{2}$ model.

This paper is organized as follows. Section II gives details of the computational scheme used to develop the grid LS method. Section III presents a demonstration of our method, in which we use the scheme for jellium electrode models to examine the transport properties of $\mathrm{Si}-\mathrm{SiO}_{2}$ and $\mathrm{Ge}-\mathrm{GeO}_{2}$ models and to reveal how the leakage current is influenced by the DB state that arises due to an oxygen vacancy. Conclusions are given in Sec. IV and mathematical details are described in Appendixes $\mathrm{A}$ and $\mathrm{B}$.

\section{COMPUTATIONAL FORMALISM}

We propose an efficient procedure to obtain the solution of the Kohn-Sham equation for a system where the nanoscale junction is sandwiched between semi-infinite electrodes within the framework of the RSFD scheme. The effective potential is close to the periodic bulk potentials, as it goes deeply inside the left and right electrodes, so that the whole infinite system can be appropriately divided into three parts: the left electrode, the transition region, and the right electrode. The Hamiltonian of the system, $H$, is defined by

$$
H=-\frac{1}{2} \nabla^{2}+v\left(\boldsymbol{r}, \boldsymbol{r}^{\prime}\right)
$$

with

$$
v\left(\boldsymbol{r}, \boldsymbol{r}^{\prime}\right)=\left[v_{\mathrm{H}}(\boldsymbol{r})+v_{\mathrm{xc}}(\boldsymbol{r})+v_{\mathrm{l}}(\boldsymbol{r})\right] \delta\left(\boldsymbol{r}-\boldsymbol{r}^{\prime}\right)+v_{\mathrm{nl}}\left(\boldsymbol{r}, \boldsymbol{r}^{\prime}\right),
$$

where $v_{\mathrm{H}}(\boldsymbol{r})$ and $v_{\mathrm{xc}}(\boldsymbol{r})$ are the Hartree and exchangecorrelation potentials, respectively, and $v_{1}(\boldsymbol{r})$ and $v_{\mathrm{nl}}\left(\boldsymbol{r}, \boldsymbol{r}^{\prime}\right)$ are the local and nonlocal parts of the atomic pseudopotentials, respectively.

Assuming that the Hamiltonian in the transition region can be decomposed into an unperturbed part $H^{0}$ and a perturbation $\delta v\left(\boldsymbol{r}, \boldsymbol{r}^{\prime}\right)=H-H^{0}$, we rewrite the Kohn-Sham equation as

$$
\left(E-H^{0}\right) \psi(\boldsymbol{r})=\int_{-\infty}^{\infty} d \boldsymbol{r}^{\prime} \delta v\left(\boldsymbol{r}, \boldsymbol{r}^{\prime}\right) \psi\left(\boldsymbol{r}^{\prime}\right),
$$

where $\psi(\boldsymbol{r})$ is the scattering wave function for an incident wave coming from the left or right electrode with energy $E$. The subscript 0 on the variables indicates that they are evaluated in the unperturbed reference system. Here, $\delta v\left(\boldsymbol{r}, \boldsymbol{r}^{\prime}\right)$ is specified to be 0 and $\psi(\boldsymbol{r})=\psi^{0}(\boldsymbol{r})$ outside the transition region by choosing the structures in each electrode region of the unperturbed reference system that are the same as those in the perturbed system. Once the retarded Green's function $g_{T}^{r 0}\left(\boldsymbol{r}, \boldsymbol{r}^{\prime} ; E\right)$ in the transition region associated with the unperturbed part $H^{0}$ is known, Eq. (3) is put into the LS equation in a form of the integral equation; i.e.,

$$
\psi(\boldsymbol{r})=\psi^{0}(\boldsymbol{r})+\iint d \boldsymbol{r}^{\prime} d \boldsymbol{r}^{\prime \prime} g_{T}^{r 0}\left(\boldsymbol{r}, \boldsymbol{r}^{\prime} ; E\right) \delta v\left(\boldsymbol{r}^{\prime}, \boldsymbol{r}^{\prime \prime}\right) \psi\left(\boldsymbol{r}^{\prime \prime}\right),
$$

with the unperturbed wave function $\psi^{0}(\boldsymbol{r})$. Equation (4) provides a unified treatment of the Kohn-Sham equation and the boundary conditions [1-8]. In the case where the incident Bloch wave $\phi^{\text {in }}\left(\boldsymbol{r}_{\|}, z\right)$ propagates from deep inside the left electrode, the boundary condition is

$$
\begin{aligned}
& \psi\left(\boldsymbol{r}_{\|}, z\right) \\
& = \begin{cases}\phi^{\text {in }}\left(\boldsymbol{r}_{\|}, z\right)+\sum_{j=1}^{N} r_{j} \phi_{j}^{\mathrm{ref}}\left(\boldsymbol{r}_{\|}, z\right) & \text { in the left electrode, } \\
\sum_{j=1}^{N} t_{j} \phi_{j}^{\mathrm{tra}}\left(\boldsymbol{r}_{\|}, z\right) & \text { in the right electrode. }\end{cases}
\end{aligned}
$$

Here, $\phi_{j}^{\text {ref }}\left(\boldsymbol{r}_{\|}, z\right)$ is a reflected wave that propagates and decays into the left electrode, $\phi_{j}^{\text {tra }}\left(\boldsymbol{r}_{\|}, z\right)$ within the right electrode is a transmitted wave, and $r_{j}$ and $t_{j}$ are unknown reflection and transmission coefficients, respectively. The lateral ( $x$ and $y$ ) and $z$ directions are set to be parallel and perpendicular to the electrode surface, respectively. The system is assumed to be periodic in the lateral direction and infinite in the $z$ direction. The case of incident electrons coming from the right electrode can be considered in the same manner.

In this paper, the LS equation is solved within the RSFD scheme [10]. The RSFD approach enables us to treat arbitrary boundary conditions and to calculate the atomic and electronic structures with a high accuracy. The whole system is composed of the transition region sandwiched between semi-infinite left and right electrodes and is divided by grid points with an equal spacing of $h_{\mu}=L_{\mu} / N_{\mu}$, where $L_{\mu}$ and $N_{\mu}$ are the length and the number of grid points in the $\mu$ direction $(\mu=x, y$, and $z$ ) of the transition region, respectively. Here, we assume a twodimensional periodicity in the lateral directions and employ a generalized $z$ coordinate $\zeta_{k}$ instead of $z_{k}$, which stands for the group index of $z$ coordinates within the closed interval $\left[z_{(k-1) \mathcal{N}_{f}+1}, z_{k \mathcal{N}_{f}}\right]$, where $\mathcal{N}_{f}$ is the number of $x-y$ grid planes involved in $\zeta_{k}$ (see Fig. 1); $\mathcal{N}_{f}$ corresponds to the order of the finite-difference approximation for the kinetic energy operator in the Kohn-Sham equation [22,23] and is chosen so as to include the nonlocal region of pseudopotentials in the cases of crystalline electrodes to obtain highly accurate results (see Sec. II B). 


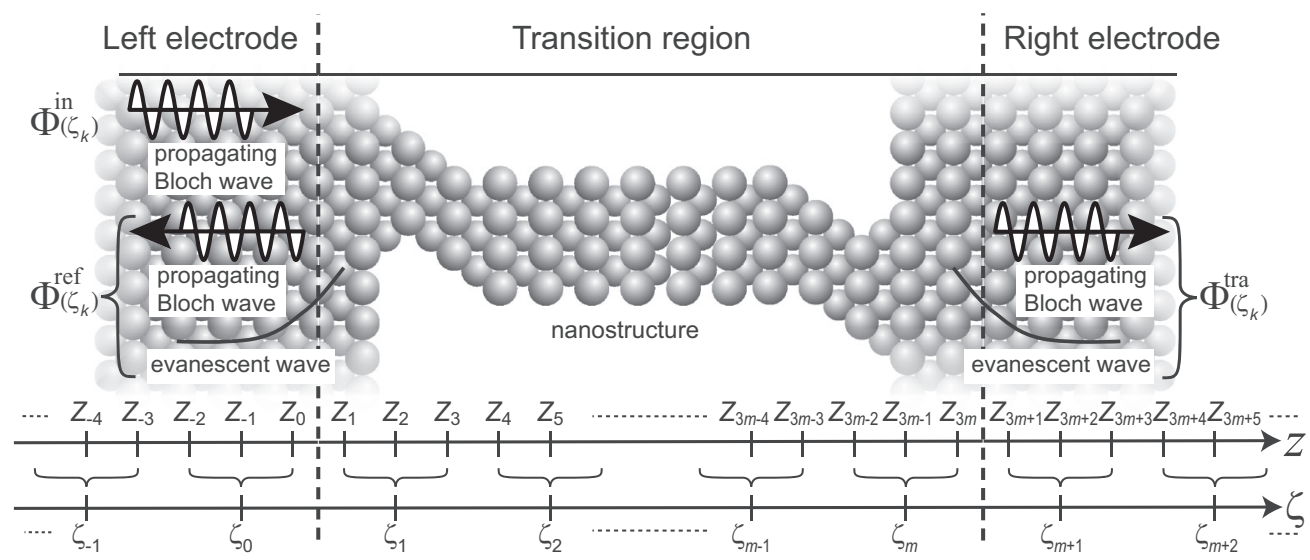

FIG. 1. Sketch of the relationship between $z$ and $\zeta$ in the computational model in the case of $\mathcal{N}_{f}=3$. The system consists of the transition region sandwiched between the left and the right semi-infinite crystalline electrodes. In the left electrode, the incident wave and the reflected waves consisting of the propagating and evanescent ones are illustrated by $\Phi^{\mathrm{in}}\left(\zeta_{k}\right)$ and $\Phi^{\text {ref }}\left(\zeta_{k}\right)$, respectively, while in the right electrode, the transmitted waves composed of the propagating and decaying evanescent ones toward the right side are denoted $\Phi^{\text {tra }}\left(\zeta_{k}\right)$. Here, $x(y)$ and $z$ are coordinates perpendicular and parallel to the nanoscale junction, respectively.

The LS equation is written in the discretized form as

$$
\begin{aligned}
\Psi\left(\zeta_{k}\right)= & \Psi^{0}\left(\zeta_{k}\right)+\sum_{l, l^{\prime}=1}^{m} G_{T}^{r 0}\left(\zeta_{k}, \zeta_{l}\right) \delta V\left(\zeta_{l}, \zeta_{l^{\prime}}\right) \Psi\left(\zeta_{l^{\prime}}\right) \\
& (k=0,1, \ldots, m+1),
\end{aligned}
$$

which is referred to as the grid LS equation, where $\Psi\left(\zeta_{k}\right)\left(\Psi^{0}\left(\zeta_{k}\right)\right)$ is a set of the $N\left(=N_{x} \times N_{y} \times \mathcal{N}_{f}\right)$ values of the wave functions on the $x-y$ planes at $\zeta=\zeta_{k}$ in the perturbed (unperturbed) system and $G_{T}^{r 0}\left(\zeta_{k}, \zeta_{l} ; E\right)$ is the discretized retarded Green's function in the unperturbed transition region.

$$
\Psi\left(\zeta_{k}\right)=\left\{\begin{array}{l}
\Phi^{\mathrm{in}}\left(\zeta_{k}\right)+\sum_{j=1}^{N} r_{j} \Phi_{j}^{\mathrm{ref}}\left(\zeta_{k}\right) \\
\sum_{j=1}^{N} t_{j} \Phi_{j}^{\mathrm{tra}}\left(\zeta_{k}\right)
\end{array}\right.
$$

The Hamiltonian matrix of the unperturbed reference system, $\hat{H}^{0}$, is taken to be a block-tridiagonal form constituted of the $N$-dimensional block matrices, $H^{0}\left(\zeta_{k}\right)$. The block matrix $G_{T}^{r 0}\left(\zeta_{k}, \zeta_{l} ; E\right)$, which is a component of the retarded Green's function matrix $\hat{G}_{T}^{r 0}=\left(E-\hat{H}^{0}\right)^{-1}$, is expressed in terms of the scattering wave functions in a variable-separable form as (see Appendix A)

$$
G_{T}^{r 0}\left(\zeta_{k}, \zeta_{l} ; E\right)= \begin{cases}U_{R}^{0}\left(\zeta_{k}\right)\left(U_{R}^{0}\left(\zeta_{l}\right)\right)^{-1} D_{l}^{0} & (k<l), \\ D_{l}^{0} & (k=l), \\ U_{L}^{0}\left(\zeta_{k}\right)\left(U_{L}^{0}\left(\zeta_{l}\right)\right)^{-1} D_{l}^{0} & (k>l) .\end{cases}
$$

Here, $U_{R}^{0}\left(\zeta_{k}\right)\left[U_{L}^{0}\left(\zeta_{k}\right)\right]$ is the $N$-dimensional matrix made up of the solutions of the Kohn-Sham equation in the case of electrons coming from the right (left) electrode in the reference
For simplicity, the notations with respect to the energy $E$, the lateral coordinates $\boldsymbol{r}_{\|, j}$, and the lateral Bloch wave vectors within the first Brillouin zone, $\boldsymbol{k}_{\|}^{B}=\left(k_{x}^{B}, k_{y}^{B}\right)$, are ignored in Eq. (6). Here, we assume that the perturbed Hamiltonian in the transition region $\left(\zeta_{1} \leqslant \zeta_{k(l)} \leqslant \zeta_{m}\right)$ can be decomposed into an unperturbed part $H^{0}\left(\zeta_{k}\right)$ and a perturbation $\delta V\left(\zeta_{k}, \zeta_{l}\right)$, in which $\delta V\left(\zeta_{k}, \zeta_{l}\right)$ has nonzero elements only in the transition region, by choosing the electrode regions to be exactly those in the perturbed system, as well as in the case of the nondiscretized treatment mentioned above. This discretized form within the framework of the RSFD approach unifies the Kohn-Sham equation and the scattering boundary conditions. The boundary condition, Eq. (5), now reads as

in the left electrode $(k \leqslant 0)$,

in the right electrode $(k \geqslant m+1)$.

system; that is,

$$
\begin{aligned}
& U_{R}^{0}\left(\zeta_{k}\right)=\left(\Psi_{R, 1}^{0}\left(\zeta_{k}\right), \Psi_{R, 2}^{0}\left(\zeta_{k}\right), \ldots, \Psi_{R, N}^{0}\left(\zeta_{k}\right)\right), \\
& U_{L}^{0}\left(\zeta_{k}\right)=\left(\Psi_{L, 1}^{0}\left(\zeta_{k}\right), \Psi_{L, 2}^{0}\left(\zeta_{k}\right), \ldots, \Psi_{L, N}^{0}\left(\zeta_{k}\right)\right),
\end{aligned}
$$

where the $N$-dimensional columnar vector $\Psi_{R, j}^{0}\left(\zeta_{k}\right)\left(\Psi_{L, j}^{0}\left(\zeta_{k}\right)\right)$ denotes the scattering wave functions at $\zeta_{k}$ for the $j$ th incident wave $\Phi_{R, j}^{0, \text { in }}\left(\Phi_{L, j}^{0, \text { in }}\right)$ incoming from deep inside the right (left) electrode, where the incident wave is considered to include an evanescent wave as well as an ordinary propagating wave; more precisely, $\left\{\Phi_{R, j}^{0, \text { in }}\right\}\left(\left\{\Phi_{L, j}^{0, \text { in }}\right\}\right)$ is taken to be a set of the $N$ generalized Bloch states consisting of leftward (rightward-) -propagating Bloch waves and decaying evanescent waves toward the left (right) side, which are the solutions of the $2 N$-dimensional generalized eigenvalue equation $[9,10,18]$. 
The matrix $D_{k}^{0}$ stands for the diagonal block-matrix element of the retarded Green's function matrix, $G_{T}^{r 0}\left(\zeta_{k}, \zeta_{k} ; E\right)$, a representation of which is derived in Appendix A as [see Eq. (A10)]

$$
\begin{aligned}
D_{k}^{0}= & {\left[-B^{0}\left(\zeta_{k-1}\right)^{\dagger} U_{R}^{0}\left(\zeta_{k-1}\right)\left(U_{R}^{0}\left(\zeta_{k}\right)\right)^{-1}+A^{0}\left(\zeta_{k}\right)\right.} \\
& \left.-B^{0}\left(\zeta_{k}\right) U_{L}^{0}\left(\zeta_{k+1}\right)\left(U_{L}^{0}\left(\zeta_{k}\right)\right)^{-1}\right]^{-1}
\end{aligned}
$$

with $A^{0}\left(\zeta_{k}\right)\left(-B^{0}\left(\zeta_{k}\right)\right)$ being the diagonal (off-diagonal) block-matrix element of $\left(E-\hat{H}^{0}\right)$.
Since $U_{R(L)}^{0}\left(\zeta_{k}\right)$ includes the exponentially growing or decaying evanescent waves, the calculation using Eq. (8) frequently gives rise to serious numerical errors [8]. We provide a remedy for this problem as follows.

Introducing the ratio matrices $X_{k}^{0}$ and $Y_{k}^{0}$ at two successive $\zeta_{k}$ points, which are defined as

$$
\begin{aligned}
& X_{k}^{0} \equiv U_{R}^{0}\left(\zeta_{k-1}\right)\left(U_{R}^{0}\left(\zeta_{k}\right)\right)^{-1}, \\
& Y_{k}^{0} \equiv U_{L}^{0}\left(\zeta_{k+1}\right)\left(U_{L}^{0}\left(\zeta_{k}\right)\right)^{-1},
\end{aligned}
$$

respectively, we obtain the following $(m+2)$-dimensional block-matrix expression for the retarded Green's function, Eq. (8):

$$
\hat{G}_{T}^{r 0}=\left[\begin{array}{ccccc}
D_{0}^{0} & X_{1}^{0} D_{1}^{0} & X_{1}^{0} X_{2}^{0} D_{2}^{0} & \cdots & \left(\prod_{j=1}^{m+1} X_{j}^{0}\right) D_{m+1}^{0} \\
Y_{0}^{0} D_{0}^{0} & D_{1}^{0} & X_{2}^{0} D_{2}^{0} & \cdots & \left(\prod_{j=2}^{m+1} X_{j}^{0}\right) D_{m+1}^{0} \\
\vdots & \vdots & \vdots & \ddots & \vdots \\
\left(\prod_{j=m}^{0} Y_{j}^{0}\right) D_{0}^{0} & \left(\prod_{j=m}^{1} Y_{j}^{0}\right) D_{1}^{0} & \left(\prod_{j=m}^{2} Y_{j}^{0}\right) D_{2}^{0} & \cdots & D_{m+1}^{0}
\end{array}\right] \text {; }
$$

that is, we rewrite the block-matrix element of $\hat{G}_{T}^{r 0}$ in Eq. (8)

as

$$
G_{T}^{r 0}\left(\zeta_{k}, \zeta_{l} ; E\right)= \begin{cases}\left(\prod_{j=k+1}^{l} X_{j}^{0}\right) D_{l}^{0} & (k<l), \\ D_{l}^{0} & (k=l), \\ \left(\prod_{j=k-1}^{l} Y_{j}^{0}\right) D_{l}^{0} & (k>l),\end{cases}
$$

and from Eqs. (11)-(13) the diagonal block-matrix element $D_{k}^{0}$ reads as

$$
D_{k}^{0}=\left[-B^{0}\left(\zeta_{k-1}\right)^{\dagger} X_{k}^{0}+A^{0}\left(\zeta_{k}\right)-B^{0}\left(\zeta_{k}\right) Y_{k}^{0}\right]^{-1}
$$

In the following subsections (Secs. II A and II B), we report efficient numerical calculation techniques for the ratio matrices $\left\{X_{k}^{0}\right\}$ and $\left\{Y_{k}^{0}\right\}$ without employing the matrices $\left\{U_{R}^{0}\left(\zeta_{k}\right)\right\}$ and $\left\{U_{L}^{0}\left(\zeta_{k}\right)\right\}$, which include evanescent waves explicitly. Our previous study [8] verified that the introduction of a ratio expression such as Eqs. (12)-(15) into the retarded Green's function enables us to avoid the numerical collapse originating from the appearance of the rapidly growing and decaying evanescent waves. By contrast, in LS simulations of electron transport through long conductor systems using the conventional Green's function in a variable-separable form, the numerical collapse is inevitable.

In the solving of Eq. (6) using an iterative method such as the conjugate gradient method, the operation of $\sum G_{T}^{r 0}\left(\zeta_{k}, \zeta_{l}\right) \delta V\left(\zeta_{l}, \zeta_{l^{\prime}}\right) \Psi\left(\zeta_{l^{\prime}}\right)$ in Eq. (6) is carried out as follows:

$$
\begin{aligned}
& \sum_{l, l^{\prime}=1}^{m} G_{T}^{r 0}\left(\zeta_{k}, \zeta_{l}\right) \delta V\left(\zeta_{l}, \zeta_{l^{\prime}}\right) \Psi\left(\zeta_{l^{\prime}}\right) \\
& \quad=\Psi^{\prime}\left(\zeta_{k}\right)+P_{L}\left(\zeta_{k}\right)+P_{R}\left(\zeta_{k}\right) \quad(k=0,1, \ldots, m+1)
\end{aligned}
$$

where

$$
\begin{aligned}
\Psi^{\prime}\left(\zeta_{k}\right)= & D_{k}^{0} \sum_{l=1}^{m} \delta V\left(\zeta_{k}, \zeta_{l}\right) \Psi\left(\zeta_{l}\right) \\
P_{L}\left(\zeta_{k}\right)= & \left(\prod_{j=k-1}^{0} Y_{j}^{0}\right) \Psi^{\prime}\left(\zeta_{0}\right)+\left(\prod_{j=k-1}^{1} Y_{j}^{0}\right) \Psi^{\prime}\left(\zeta_{1}\right) \\
& +\cdots+Y_{k-1}^{0} \Psi^{\prime}\left(\zeta_{k-1}\right) \\
P_{R}\left(\zeta_{k}\right)= & X_{k+1}^{0} \Psi^{\prime}\left(\zeta_{k+1}\right)+X_{k+1}^{0} X_{k+2}^{0} \Psi^{\prime}\left(\zeta_{k+2}\right) \\
& +\cdots+\left(\prod_{j=k+1}^{m+1} X_{j}^{0}\right) \Psi^{\prime}\left(\zeta_{m+1}\right)
\end{aligned}
$$


It is easily shown that the sequences $\left\{P_{L}\left(\zeta_{k}\right)\right\}$ and $\left\{P_{R}\left(\zeta_{k}\right)\right\}$ satisfy the following recursive relations:

$$
\begin{aligned}
& P_{L}\left(\zeta_{k}\right)= \begin{cases}0 & (k=0,1), \\
Y_{k-1}^{0}\left(P_{L}\left(\zeta_{k-1}\right)+\Psi^{\prime}\left(\zeta_{k-1}\right)\right) & (k=2,3, \ldots, m+1) ;\end{cases} \\
& P_{R}\left(\zeta_{k}\right)= \begin{cases}0 & (k=m+1, m), \\
X_{k+1}^{0}\left(P_{R}\left(\zeta_{k+1}\right)+\Psi^{\prime}\left(\zeta_{k+1}\right)\right) & (k=m-1, \ldots, 1,0) .\end{cases}
\end{aligned}
$$

Here, we have used the fact that $\delta V\left(\zeta_{k}, \zeta_{l}\right)=0$ outside the transition region of $\zeta_{1} \leqslant \zeta_{k(l)} \leqslant \zeta_{m}$. It should be emphasized that since the elements of $\hat{G}_{T}^{r 0}$ in Eq. (15) are no longer in a variable-separable form, the amount of $[(m+2) N]^{2}$ for each multiplication is expected to be required; nevertheless, it is reduced to the order of $(m+2) N^{2}$ by virtue of Eqs. (21) and (22), which means that the present method does not suffer from numerical collapse without increasing the computational cost.

\section{A. Jellium electrodes}

The case in which the electrodes are approximated by structureless jellium models is treated. The jellium electrode approximation has been successfully applied to the interpretation of electron-transport properties with a lower computational load [5,24-30]. A free electron system is chosen as the unperturbed one with the Hamiltonian $H^{0}$, where a completely flat potential is assumed, for simplicity. In this case, all nonlocal parts of the pseudopotentials are contained in the perturbation term and the retarded Green's function in the free electron system is required, which is more conveniently described using $z_{k}$ instead of $\zeta_{k}$. Therefore, any $\mathcal{N}_{f}$ can be adopted to satisfy the grid LS procedure. In Appendix B, we discuss the analytical expression of the Green's function in terms of $z_{k}$ in a general $\mathcal{N}_{f}$ case.

We here give details on the implementation of the analytically expressed retarded Green's function in the threedimensional central finite-difference $\left(\mathcal{N}_{f}=1\right)$ case, for example, which is written as

$$
G_{T}^{r 0}\left(\boldsymbol{r}_{\|, j}, z_{k}, \boldsymbol{r}_{\|, j^{\prime}}, z_{l} ; E\right)=\frac{h_{z}^{2}}{i N} \sum_{n_{x}=-\frac{N_{x}-1}{2}}^{\frac{N_{x}-1}{2}} \sum_{n_{y}=-\frac{N_{y}-1}{2}}^{\frac{N_{y}-1}{2}} \frac{\exp \left[i\left(\boldsymbol{G}_{\|, n}+\boldsymbol{k}_{\|}^{B}\right) \cdot\left(\boldsymbol{r}_{\|, j}-\boldsymbol{r}_{\|, j^{\prime}}\right)+i \mathcal{K}_{1}\left|z_{k}-z_{l}\right|\right]}{\sin \mathcal{K}_{1} h_{z}} .
$$

Here,

$$
\boldsymbol{G}_{\|, n}=\left(G_{n_{x}}, G_{n_{y}}\right)=\left(\frac{2 \pi}{h_{x} N_{x}} n_{x}, \frac{2 \pi}{h_{y} N_{y}} n_{y}\right),
$$

$\boldsymbol{r}_{\|, j}=\left(x_{j_{x}}, y_{j_{y}}\right)$ are the lateral coordinates with $j_{x(y)}=1,2, \ldots, N_{x(y)}\left[N_{x(y)}\right.$ is chosen an odd integer for convenience $]$, and

$$
\mathcal{K}_{1}= \begin{cases}\frac{1}{h_{z}} \cos ^{-1}\left[1-h_{z}^{2}\left(E-E_{n_{x}, n_{y}}^{\left(\mathcal{N}_{f}=1\right)}\right)\right] & \ldots E_{n_{x}, n_{y}}^{\left(\mathcal{N}_{f}=1\right)} \leqslant E<E_{n_{x}, n_{y}}^{\left(\mathcal{N}_{f}=1\right)}+\frac{2}{h_{z}^{2}}, \\ i \frac{1}{h_{z}} \cosh ^{-1}\left[1-h_{z}^{2}\left(E-E_{n_{x}, n_{y}}^{\left(\mathcal{N}_{f}=1\right)}\right)\right] & \ldots E<E_{n_{x}, n_{y}}^{\left(\mathcal{N}_{f}=1\right)} \\ \frac{1}{h_{z}}\left[\pi+i \cosh ^{-1}\left[-1+h_{z}^{2}\left(E-E_{n_{x}, n_{y}}^{\left(\mathcal{N}_{f}=1\right)}\right)\right]\right] & \ldots E_{n_{x}, n_{y}}^{\left(\mathcal{N}_{f}=1\right)}+\frac{2}{h_{z}^{2}} \leqslant E,\end{cases}
$$

with

$$
E_{n_{x}, n_{y}}^{\left(\mathcal{N}_{f}=1\right)}=\frac{1}{h_{x}^{2}}\left[1-\cos \left(G_{n_{x}}+k_{x}^{B}\right) h_{x}\right]+\frac{1}{h_{y}^{2}}\left[1-\cos \left(G_{n_{y}}+k_{y}^{B}\right) h_{y}\right] .
$$

In the derivation of Eqs. (23)-(26), we used Eqs. (B22) and (B23) and the extension of Eq. (B27) to the case of the threedimensional space.

Since $D_{k}^{0}$ defined by Eq. (15) is the diagonal block-matrix element of the retarded Green's function $G_{T}^{r 0}\left(z_{k}, z_{l} ; E\right)$, the $j$ th row and $j^{\prime}$ th column element $\left(D_{k}^{0}\right)_{j, j^{\prime}}$ is expressed as

$$
\left(D_{k}^{0}\right)_{j, j^{\prime}} \equiv G_{T}^{r 0}\left(\boldsymbol{r}_{\|, j}, z_{k}, \boldsymbol{r}_{\|, j^{\prime}}, z_{k} ; E\right)=\frac{h_{z}^{2}}{i N} \sum_{n_{x}=-\frac{N_{x}-1}{2}}^{\frac{N_{x}-1}{2}} \sum_{n_{y}=-\frac{N_{y}-1}{2}}^{\frac{N_{y}-1}{2}} \exp \left[i\left(\boldsymbol{G}_{\|, n}+\boldsymbol{k}_{\|}^{B}\right) \cdot\left(\boldsymbol{r}_{\|, j}-\boldsymbol{r}_{\|, j^{\prime}}\right)\right] \frac{1}{\sin \mathcal{K}_{1} h_{z}} .
$$


One can see from Eq. (27) that $\left(D_{k}^{0}\right)_{j, j^{\prime}}$, and thus $D_{k}^{0}$, is $k$ independent, owing to the translation invariance in the $z$ direction. On the other hand, by Eq. (15), $X_{k}^{0}$ and $Y_{k}^{0}$ are given by

$$
\begin{aligned}
& X_{k}^{0}=G_{T}^{r 0}\left(z_{k-1}, z_{k} ; E\right)\left(D_{k}^{0}\right)^{-1}, \\
& Y_{k}^{0}=G_{T}^{r 0}\left(z_{k+1}, z_{k} ; E\right)\left(D_{k}^{0}\right)^{-1},
\end{aligned}
$$

and from Eq. (23), the $j$ th row and $j^{\prime}$ th column matrix element of $G_{T}^{r 0}\left(z_{k \pm 1}, z_{k} ; E\right)$ are described as

$$
\begin{aligned}
\left(G_{T}^{r 0}\left(z_{k \pm 1}, z_{k} ; E\right)\right)_{j, j^{\prime}} & \equiv G_{T}^{r 0}\left(\boldsymbol{r}_{\|, j}, z_{k \pm 1}, \boldsymbol{r}_{\|, j^{\prime}}, z_{k} ; E\right) \\
& =\frac{h_{z}^{2}}{i N} \sum_{n_{x}=-\frac{N_{x}-1}{2}}^{\frac{N_{x}-1}{2}} \sum_{n_{y}=-\frac{N_{y}-1}{2}}^{\frac{N_{y}-1}{2}} \exp \left[i\left(\boldsymbol{G}_{\|, n}+\boldsymbol{k}_{\|}^{B}\right) \cdot\left(\boldsymbol{r}_{\|, j}-\boldsymbol{r}_{\|, j^{\prime}}\right)\right] \frac{1}{\sin \mathcal{K}_{1} h_{z}} \exp \left(i \mathcal{K}_{1} h_{z}\right) .
\end{aligned}
$$

This implies that $X_{k}^{0}$ and $Y_{k}^{0}$ are also $k$ independent and

$$
X_{k}^{0}=Y_{k}^{0}
$$

After some calculations,

$$
\begin{aligned}
\left(X_{k}^{0}\right)_{j, j^{\prime}} & \equiv\left[G_{T}^{r 0}\left(z_{k-1}, z_{k} ; E\right)\left(D_{k}^{0}\right)^{-1}\right]_{j, j^{\prime}} \\
& =\frac{1}{N} \sum_{n_{x}=-\frac{N_{x}-1}{2}}^{\frac{N_{x}-1}{2}} \sum_{n_{y}=-\frac{N_{y}-1}{2}}^{\frac{N_{y}-1}{2}} \exp \left[i\left(\boldsymbol{G}_{\|, n}+\boldsymbol{k}_{\|}^{B}\right) \cdot\left(\boldsymbol{r}_{\|, j}-\boldsymbol{r}_{\|, j^{\prime}}\right)\right] \exp \left(i \mathcal{K}_{1} h_{z}\right)
\end{aligned}
$$

is obtained [31]. Hereafter, $D_{k}^{0}$ and $X_{k}^{0}$ are denoted $D^{0}$ and $X^{0}$, respectively, since they are $k$ independent.

The products of the matrix $D^{0}\left(X^{0}\right)$ and vectors $\left\{f\left(\boldsymbol{r}_{\|, j}, z_{k}\right) \mid j=1,2, \ldots, N\right\}$ as required in the computations of Eqs. (18), (21), and (22) can be easily carried out in the momentum space, since they are written in the convolution form of the two-dimensional discrete Fourier transform. Owing to the orthogonality of the plane waves, the Fourier-transformed $D^{0}$ and $X^{0}$ are represented as the diagonalized matrices; i.e.,

$$
\begin{aligned}
\mathcal{F}\left[D^{0}\right]_{n, n^{\prime}} & \equiv \frac{1}{N} \sum_{\boldsymbol{r}_{\|, j}} \sum_{\boldsymbol{r}_{\|, j^{\prime}}} \exp \left[-i\left(\boldsymbol{G}_{\|, n}+\boldsymbol{k}_{\|}^{B}\right) \cdot \boldsymbol{r}_{\|, j}+i\left(\boldsymbol{G}_{\|, n^{\prime}}+\boldsymbol{k}_{\|}^{B}\right) \cdot \boldsymbol{r}_{\|, j^{\prime}}\right]\left(D^{0}\right)_{j, j^{\prime}} \\
& =-i h_{z}^{2} \delta_{n n^{\prime}} \frac{1}{\sin \left(\mathcal{K}_{1} h_{z}\right)}, \\
\mathcal{F}\left[X^{0}\right]_{n, n^{\prime}} & \equiv \frac{1}{N} \sum_{\boldsymbol{r}_{\|, j}} \sum_{\boldsymbol{r}_{\|, j^{\prime}}} \exp \left[-i\left(\boldsymbol{G}_{\|, n}+\boldsymbol{k}_{\|}^{B}\right) \cdot \boldsymbol{r}_{\|, j}+i\left(\boldsymbol{G}_{\|, n^{\prime}}+\boldsymbol{k}_{\|}^{B}\right) \cdot \boldsymbol{r}_{\|, j^{\prime}}\right]\left(X^{0}\right)_{j, j^{\prime}} \\
& =\delta_{n n^{\prime}} \exp \left(i \mathcal{K}_{1} h_{z}\right),
\end{aligned}
$$

respectively. Finally, one can obtain the matrix elements of the Fourier transform of the terms shown in Eqs. (18), (21), and (22) as

$$
\begin{aligned}
& \mathcal{F}\left[\Psi^{\prime}\left(z_{k}\right)\right]_{n}= \sum_{n^{\prime}} \mathcal{F}\left[D^{0}\right]_{n, n^{\prime}} \mathcal{F}\left[\sum_{l} \delta V\left(z_{k}, z_{l}\right) \Psi\left(z_{l}\right)\right]_{n^{\prime}}, \\
& \mathcal{F}\left[P_{L}\left(z_{k}\right)\right]_{n}= \begin{cases}0 & (k=0,1), \\
\sum_{n^{\prime}} \mathcal{F}\left[X^{0}\right]_{n, n^{\prime}}\left(\mathcal{F}\left[P_{L}\left(z_{k-1}\right)\right]_{n^{\prime}}+\mathcal{F}\left[\Psi^{\prime}\left(z_{k-1}\right)\right]_{n^{\prime}}\right) & (k=2,3, \ldots, m+1),\end{cases} \\
& \mathcal{F}\left[P_{R}\left(z_{k}\right)\right]_{n}= \begin{cases}0 & (k=m+1, m), \\
\sum_{n^{\prime}} \mathcal{F}\left[X^{0}\right]_{n, n^{\prime}}\left(\mathcal{F}\left[P_{R}\left(z_{k+1}\right)\right]_{n^{\prime}}+\mathcal{F}\left[\Psi^{\prime}\left(z_{k+1}\right)\right]_{n^{\prime}}\right) & (k=m-1, \ldots, 1,0),\end{cases}
\end{aligned}
$$

respectively.

To calculate the product in Eq. (6), the computational cost of $O\left(N_{\text {in }} \times N_{z}^{2} \times N^{2}\right)$ is required, where $N_{\text {in }}$ is the number of incident waves. However, by introducing the two-dimensional discrete fast Fourier transform algorithm, 

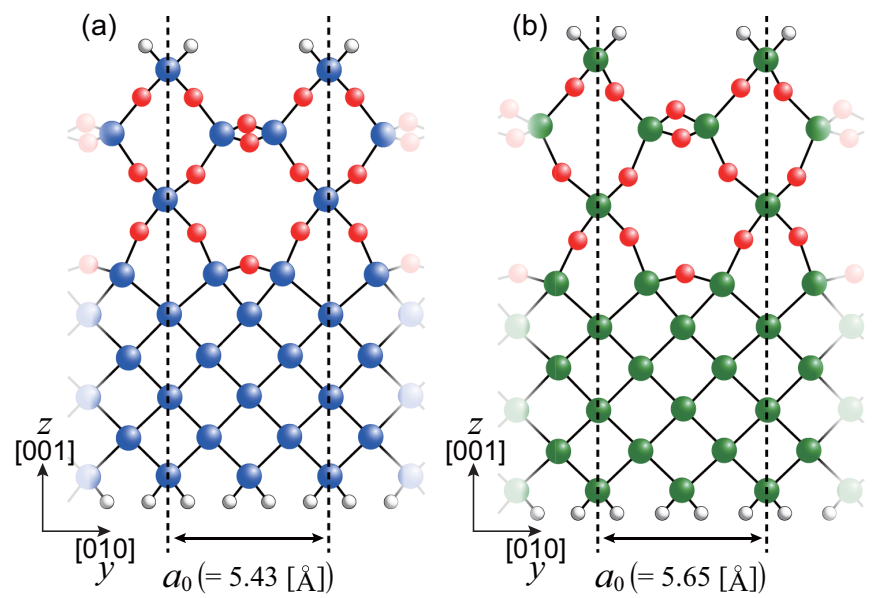

FIG. 2. (Color online) Schematics of unit cells of the (a) $\mathrm{Si}_{-} \mathrm{SiO}_{2}$ and (b) $\mathrm{Ge}-\mathrm{GeO}_{2}$ models. Dashed lines indicate the boundaries of the cell. White spheres, $\mathrm{H}$ atoms; blue spheres, $\mathrm{Si}$ atoms; green spheres, $\mathrm{Ge}$ atoms; and red spheres, $\mathrm{O}$ atoms.

the cost of the product shown in Eqs. (35)-(37) decreases to $O\left(N_{\text {in }} \times N_{z} \times N \log N\right)$, since the off-diagonal elements of the Fourier-transformed matrices $\mathcal{F}\left[D^{0}\right]$ and $\mathcal{F}\left[X^{0}\right]$ are 0 as seen in Eqs. (33) and (34). The Fourier transform of a columnar vector and the inverse Fourier transform of $\mathcal{F}\left[D^{0}\right] \times \mathcal{F}\left[f_{k}\right]$ and $\mathcal{F}\left[X^{0}\right] \times \mathcal{F}\left[f_{k}\right]$ are carried out at each $z_{k}$ point using the fast Fourier transform algorithm. Here, $\mathcal{F}\left[f_{k}\right]$ represents the Fourier-transformed vector of $\left\{f\left(\boldsymbol{r}_{\|, j}, z_{k}\right) \mid j=1,2, \ldots, N\right\}$. Thus, the maximum order of the calculations is improved from $O\left(N_{\text {in }} \times N_{z}^{2} \times N^{2}\right)$ to $O\left(N_{\text {in }} \times N_{z} \times N \log N\right)$. The above-mentioned discussion of the central finite-difference approximation can be straightforwardly extended to cases of the higher order finite-difference approach.

\section{B. Crystalline electrodes}

A general case is discussed where a system with atomistic crystalline electrodes is chosen as the unperturbed reference system; one electrode is confronted with the other across the vacuum region. In practice, the matching $x-y$ planes between the transition and the electrode regions are set to be inside each electrode surface so that any effect of the sandwiched structures on the potential of electrode regions becomes negligible small. Here, the retarded Green's function is denoted in terms of $\zeta_{k}$. Also, $\mathcal{N}_{f}$ is determined so as to entirely cover the nonlocal regions of the pseudopotentials extending across the matching planes.

The matrices $X_{0}^{0}$ and $Y_{m+1}^{0}$ defined by Eqs. (12) and (13) are described as

$$
\begin{gathered}
X_{0}^{0} \equiv U_{R}^{0}\left(\zeta_{-1}\right)\left(U_{R}^{0}\left(\zeta_{0}\right)\right)^{-1}=\left(B^{0}\left(\zeta_{-1}\right)^{\dagger}\right)^{-1} \Sigma_{L}^{r 0}\left(\zeta_{0}\right), \\
Y_{m+1}^{0} \equiv U_{L}^{0}\left(\zeta_{m+2}\right)\left(U_{L}^{0}\left(\zeta_{m+1}\right)\right)^{-1}=\left(B^{0}\left(\zeta_{m+1}\right)\right)^{-1} \Sigma_{R}^{r 0}\left(\zeta_{m+1}\right),
\end{gathered}
$$

where $\sum_{L}^{r 0}\left(\zeta_{0}\right)\left(\sum_{R}^{r 0}\left(\zeta_{m+1}\right)\right)$ is the self-energy term defined on the left (right-)-electrode surface and can be calculated using the continued-fraction equation; for details of the derivation of Eqs. (38) and (39) and computation of the self-energy terms, see Refs. [10] and [18]. For the sake of comparison, we note that $U_{R(L)}\left(\zeta_{k}\right)$ defined by Eqs. (9) and (10) plays roles similar to those of $Q^{p(q)}\left(\zeta_{k}\right)$ of Eq. (15) in Ref. [18]. We also emphasize that the accuracy of $\Sigma_{R(L)}\left(\zeta_{k}\right)$ is enhanced by making use of the continued-fraction equation in a self-consistent manner, as shown in Eqs. (16)-(18) in Ref. [18].

It should be noted that the terms $\left\{X_{k}^{0}\right\}$ can be sequentially computed as

$$
\begin{aligned}
X_{1}^{0} & =\left(A^{0}\left(\zeta_{0}\right)-B^{0}\left(\zeta_{-1}\right)^{\dagger} X_{0}^{0}\right)^{-1} B^{0}\left(\zeta_{0}\right), \\
X_{2}^{0} & =\left(A^{0}\left(\zeta_{1}\right)-B^{0}\left(\zeta_{0}\right)^{\dagger} X_{1}^{0}\right)^{-1} B^{0}\left(\zeta_{1}\right), \\
X_{3}^{0} & =\left(A^{0}\left(\zeta_{2}\right)-B^{0}\left(\zeta_{1}\right)^{\dagger} X_{2}^{0}\right)^{-1} B^{0}\left(\zeta_{2}\right), \\
& \vdots \\
X_{m+1}^{0} & =\left(A^{0}\left(\zeta_{m}\right)-B^{0}\left(\zeta_{m-1}\right)^{\dagger} X_{m}^{0}\right)^{-1} B^{0}\left(\zeta_{m}\right),
\end{aligned}
$$

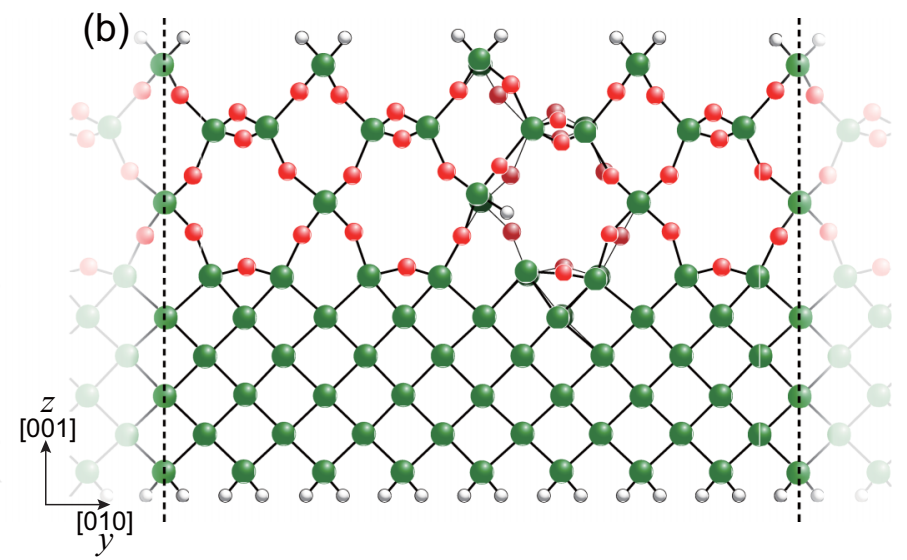

FIG. 3. (Color online) Schematis of the (a) $\mathrm{Si}-\mathrm{SiO}_{2}$ and (b) $\mathrm{Ge}-\mathrm{GeO}_{2}$ models with an oxygen vacancy after geometrical optimization. Dashed lines indicate the boundaries of supercells. Symbols are the same as in Fig. 2. 
which are easily derived from Eq. (A3), and similarly, the iterative series of $\left\{Y_{k}^{0}\right\}$ are obtainable from Eq. (A4) as

$$
\begin{aligned}
Y_{m}^{0} & =\left(A^{0}\left(\zeta_{m+1}\right)-B^{0}\left(\zeta_{m+1}\right) Y_{m+1}^{0}\right)^{-1} B^{0}\left(\zeta_{m}\right)^{\dagger}, \\
Y_{m-1}^{0} & =\left(A^{0}\left(\zeta_{m}\right)-B^{0}\left(\zeta_{m}\right) Y_{m}^{0}\right)^{-1} B^{0}\left(\zeta_{m-1}\right)^{\dagger}, \\
Y_{m-2}^{0} & =\left(A^{0}\left(\zeta_{m-1}\right)-B^{0}\left(\zeta_{m-1}\right) Y_{m-1}^{0}\right)^{-1} B^{0}\left(\zeta_{m-2}\right)^{\dagger}, \\
& \vdots \\
Y_{0}^{0} & =\left(A^{0}\left(\zeta_{1}\right)-B^{0}\left(\zeta_{1}\right) Y_{1}^{0}\right)^{-1} B^{0}\left(\zeta_{0}\right)^{\dagger}
\end{aligned}
$$

The recursive relations Eqs. (40) and (41) allow us to calculate all the matrix elements by a linear scaling operation (order- $N$ calculation procedure) at a limited computational cost. It is also noted that, using Eqs. (40) and (41), $X_{k}^{0}$ and $Y_{k}^{0}$ are stably computed without involving error accumulation since the errors due to the appearance of evanescent waves are eliminated by introducing the ratios of these waves at two successive grid points. Finally, the diagonal block-matrix element $D_{k}^{0}$ is given by Eq. (16). Once $D_{k}^{0}, X_{k}^{0}$, and $Y_{k}^{0}$ for any $k(0 \leqslant k \leqslant m+1)$ are known, all of the matrix elements of $\hat{G}_{T}^{r 0}$ in Eq. (15) are determined, and the algorithm of Eqs. (21) and (22) can be utilized.

\section{APPLICATIONS}

To demonstrate the performance of the grid LS method, we examine the electron-transport properties of models of semiconductor-insulator interfaces sandwiched between semiinfinite jellium electrodes. Recently, the germanium-based metal-oxide-semiconductor field-effect transistor has attracted significant attention because the electronic band gap of germanium $(\sim 0.66 \mathrm{eV})$ is lower than that of silicon $(\sim 1.12$ $\mathrm{eV})$, which allows for reduced operating voltages. In a highly

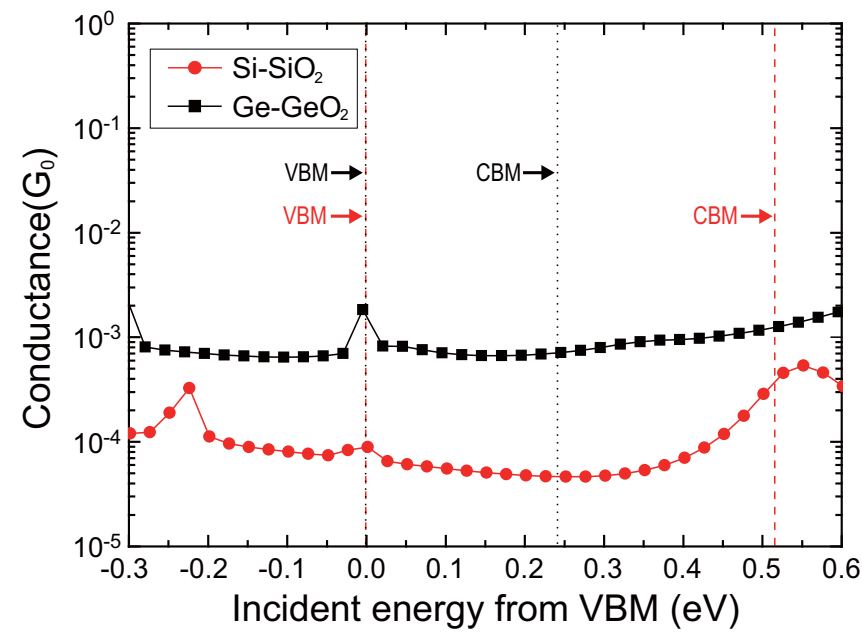

FIG. 4. (Color online) Conductance of the $\mathrm{Si}-\mathrm{SiO}_{2}$ and $\mathrm{Ge}-\mathrm{GeO}_{2}$ models as functions of the incident electron energy measured from the valence band maximum (VBM) of the substrates. The (red) circles and black squares represent conductance spectra of the $\mathrm{Si}_{-} \mathrm{SiO}_{2}$ and Ge$\mathrm{GeO}_{2}$ models, respectively. The VBM and conduction band minimum (CBM) of $\mathrm{Si}(\mathrm{Ge})$ substrate are indicated by dashed (dotted) vertical lines.

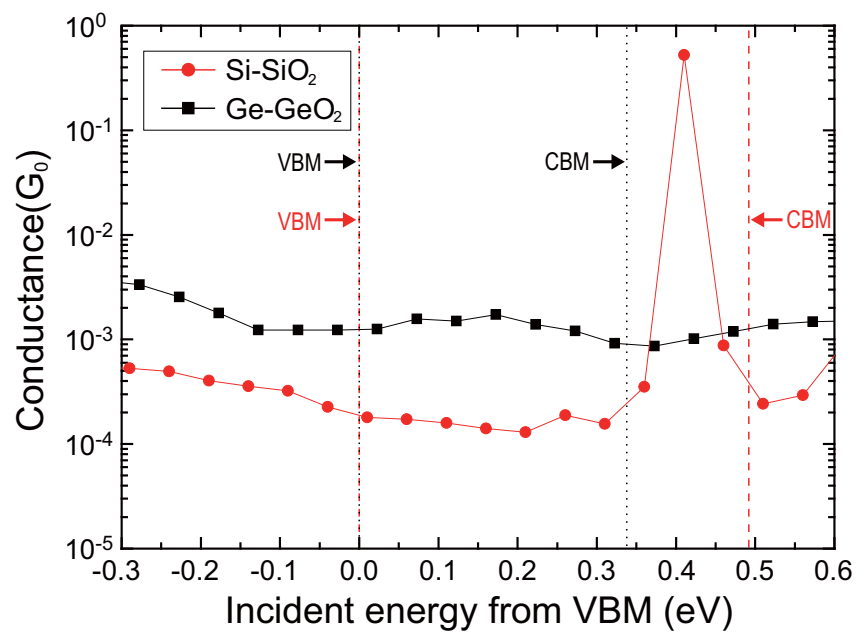

FIG. 5. (Color online) Conductance of the $\mathrm{Si}-\mathrm{SiO}_{2}$ and $\mathrm{Ge}-\mathrm{GeO}_{2}$ models as functions of the incident electron energy measured from the valence band maximum (VBM) of the substrates. Symbols are the same as in Fig. 4. The VBM and conduction band minimum (CBM) of $\mathrm{Si}(\mathrm{Ge})$ substrate are indicated by dashed (dotted) vertical lines.

integrated circuit, it is known that a large leakage current is induced by defects such as impurities and oxygen vacancies in the thin gate oxide layer. So, the relationship between the DB introduced by defects and the leakage current in $\mathrm{Si}-\mathrm{SiO}_{2}$ interfaces has been extensively investigated [32-39], while the role of the DB state in $\mathrm{Ge}-\mathrm{GeO}_{2}$ interfaces is controversial. One of the present authors (T.O.) has performed several investigations on $\mathrm{Ge}-\mathrm{GeO}_{2}$ interfaces [40-42]. In recent work, the relationship between atomic configurations and electronic structures of (001) $\mathrm{Si}_{-} \mathrm{SiO}_{2}$ and (001) Ge- $\mathrm{GeO}_{2}$ models with

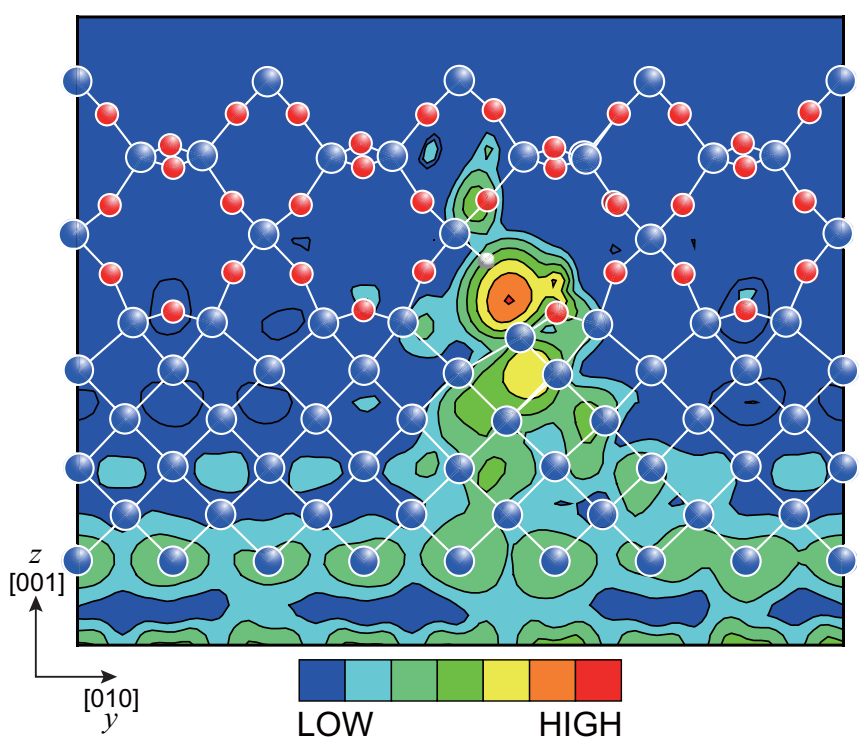

FIG. 6. (Color online) Contour plot of the charge-density distribution of electrons flowing over the $\mathrm{Si}-\mathrm{SiO}_{2}$ model with an incident energy of VBM $+0.41 \mathrm{eV}$. Symbols are the same as in Fig. 2(a). Here the charge density is integrated in the [100] $(x)$ direction. Each contour represents twice or half the density of adjacent contours; the lowest contour is $1.97 \times 10^{-4}$ electron $/ \mathrm{eV} / \AA^{2}$. 
DBs was explored using first-principles simulations within the framework of the local density approximation (LDA) [43]. It was found that the Si-DB state is located near the midgap of the $\mathrm{Si}$ substrate corresponding to the Fermi level, while the Ge-DB state lies near the top of the valence band which is 0.3 $\mathrm{eV}$ below the Fermi level [42].

To examine how DB states with different characteristics affect leakage currents, we performed transport simulations of electrons flowing across the (001) $\mathrm{Si}_{-}-\mathrm{SiO}_{2}$ and $(001) \mathrm{Ge}-$ $\mathrm{GeO}_{2}$ models. The magnitude of the leakage current flowing through insulators is so small that it can be easily affected by interactions between electrodes and interface models and by the value of the energy band gap, which is underestimated by the LDA calculation. Therefore, in this paper, we discuss qualitatively the ratio of the leakage current between models with and models without a defect.

Figure 2 illustrates a unit cell of each interface model. In these models, the side lengths of the cell in the lateral direction parallel to the interface for the $\mathrm{Si}-\mathrm{SiO}_{2}\left(\mathrm{Ge}-\mathrm{GeO}_{2}\right)$ model were taken to be the experimental lattice constant of bulk $\mathrm{Si}(\mathrm{Ge}), a_{0}=5.43(5.65) \AA$. The thicknesses of the $\mathrm{SiO}_{2}$
$\left(\mathrm{GeO}_{2}\right)$ layer and the $\mathrm{Si}(\mathrm{Ge})$ substrate were 7.34 (7.25) and 7.18 (7.37) $\AA$, respectively. In calculations for models with an oxygen vacancy, we introduced the defect into a supercell comprising $4 \times 4$ unit cells in the lateral direction (Fig. 3); this is large enough to avoid interactions between defects in neighboring cells. Two Si $(\mathrm{Ge}) \mathrm{DBs}$ were generated near the interface between the $\mathrm{Si}(\mathrm{Ge})$ substrate and the oxide layer in one unit cell by removing a bridging oxygen atom in a manner similar to that used in the previous study [42]. One of the DBs is passivated by a hydrogen atom, while the other remains with the $\mathrm{Si}(\mathrm{Ge})$ atom of the center back-bonded to two neighboring $\mathrm{Si}(\mathrm{Ge})$ atoms and an oxygen atom $\left[{ }^{\bullet} \mathrm{Si} \equiv \mathrm{Si}_{2} \mathrm{O}\right.$ $\left({ }^{\bullet} \mathrm{Ge} \equiv \mathrm{Ge}_{2} \mathrm{O}\right)$ ]. For no-defect models, the unit cell of each model, depicted in Fig. 2, was employed with $4 \times 4$ sampling $k$ points in the two-dimensional Brillouin zone for comparison with models including defects.

We first optimized the atomic and electronic structures of the models. First-principles calculations based on the RSFD approach were performed in the manner described in Ref. [42] with a grid spacing of $0.15 \AA$. The size of the supercell in the [001] $(z)$ direction was taken to be $5 a_{0}$, including a

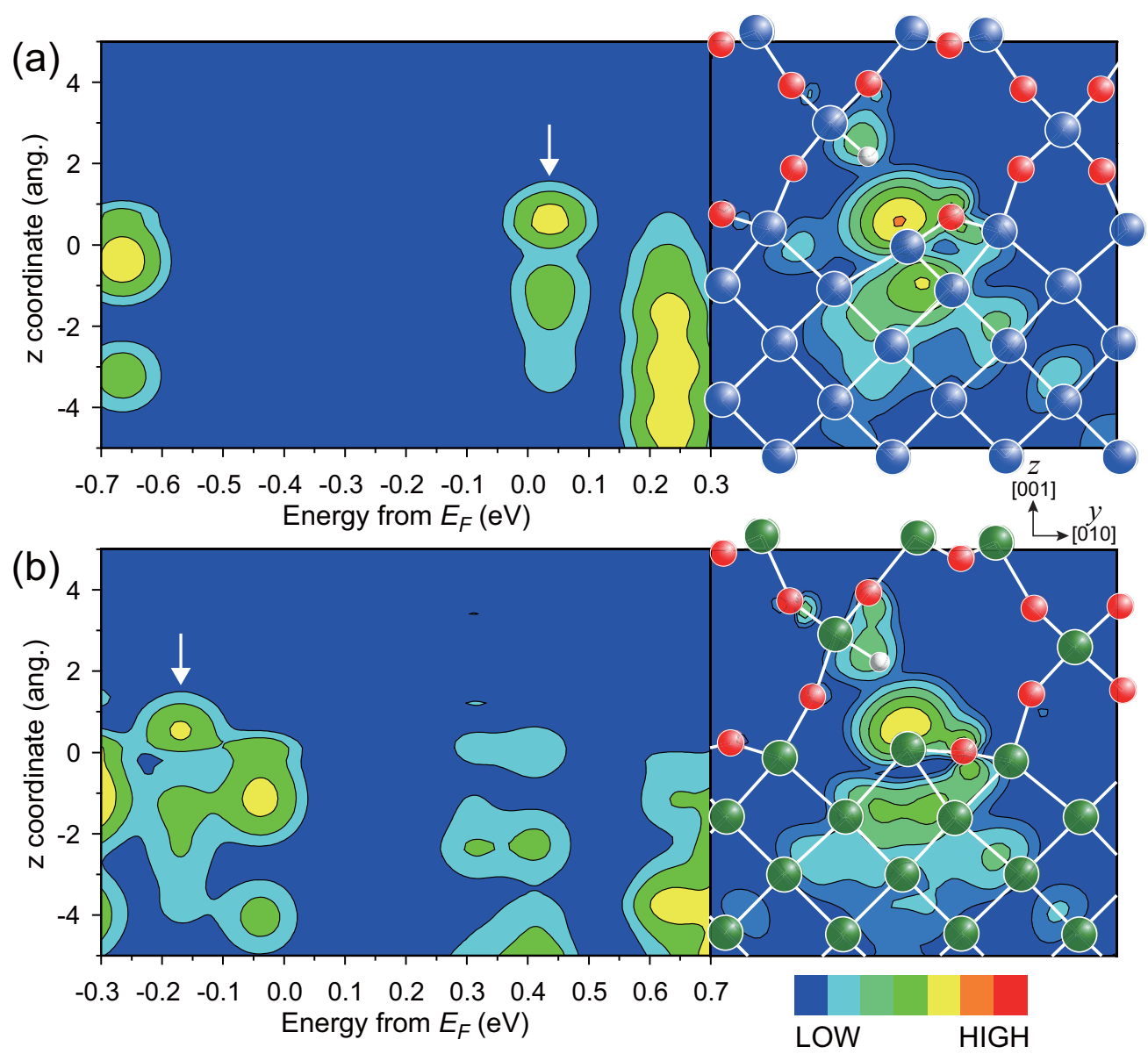

FIG. 7. (Color online) Contour plots of the density of states (DOS) (left panels) and charge density of dangling-bond (DB) states (right panels) for the (a) $\mathrm{Si}_{-} \mathrm{SiO}_{2}$ and (b) $\mathrm{Ge}-\mathrm{GeO}_{2}$ models without electrodes. Arrows denote DOS peaks derived from DB states. Energies are measured from the Fermi level $E_{F}$, and the $z$ coordinate of the atom with a DB is set to 0 . In the right panels, the charge density is integrated in the [100] $(x)$ direction, and symbolsare the same as in Fig. 2. The coordinate in the $z$ direction corresponds to that in the left panel. Each contour represents twice or half the density of adjacent contours; the lowest contour is $2.56 \times 10^{-2}$ electron $/ \mathrm{eV} / \AA\left(2.79 \times 10^{-3}\right.$ electron $\left./ \AA^{2}\right)$ in the left (right) panels. 
large enough vacuum region, and the top and bottom layers of the models were terminated by hydrogen atoms. As shown in Fig. 3, which illustrates the relaxed configurations, the Si atom with a DB is pulled down to the Si substrate, whereas the Ge atom with a DB is slightly raised toward the oxide layer.

Next, we examined the leakage currents caused by introducing oxygen vacancies into the models. Employing the optimized effective Kohn-Sham potential, we used the grid LS method to evaluate the scattering wave functions for electrons incident from the bottom-side electrode. The conductance at the limits of zero temperature and bias are described by the Landauer-Büttiker formula [44]. In the transport calculation, the top and bottom sides of each model were connected to aluminum jellium electrodes without terminating hydrogen atoms. The Wigner-Seitz radius $r_{s}$ was 2.07, which corresponds to the valence electron density of bulk aluminum.

Figure 4 shows the computed conductance spectra for the no-defect $\mathrm{Si}-\mathrm{SiO}_{2}$ and $\mathrm{Ge}-\mathrm{GeO}_{2}$ models as functions of the incident electron energy measured from the valence band maximum (VBM) of the substrate. Although some small peaks derived from the bulk states in the valence band of the Si and Ge substrates appear in Fig. 4, both models exhibit highly suppressed conductivities in the band-gap region between the VBM and the conduction band minimum (CBM) of the substrates. Figure 5 represents the conductance spectra for the $\mathrm{Si}-\mathrm{SiO}_{2}$ and $\mathrm{Ge}-\mathrm{GeO}_{2}$ models with an oxygen vacancy. No remarkable peaks appear in the spectrum of the $\mathrm{Ge}-\mathrm{GeO}_{2}$ model; however, for the $\mathrm{Si}_{-} \mathrm{SiO}_{2}$ model, a peak with high transmission occurs around $\mathrm{VBM}+0.41 \mathrm{eV}$, where electrons flow through the oxide layer via the Si-DB state as shown in the charge density distribution of the scattering electron (Fig. 6). In addition, Fig. 7 exhibits contour plots of the density of states (DOS) integrated in the lateral directions (left panels) and the charge density distributions of the DB states (right panels) for the defect-introduced models without electrodes. In Fig. 7(a), the white arrow identifies the peak in the DOS derived from the DB state between the VBM and the CBM of the Si substrate. In Fig. 7(b), the Ge-DB state is coupled to the states in the valence band of the Ge substrate. The relative positions of the VBM, CBM, and DB states are modulated when each model is connected to electrodes. When a DB appears in diamond-structured semiconductors, there are two possibilities: the DB state tends to become either more $s$ type or more $p$ type [45]. In the $p$-type DB state, the three remaining bonds tend to become $s p^{2}$ hybridized and, to reduce the strain, prefer to be in a plane. This occurs in Fig. 7(a), wherein the $\mathrm{Si}$ atom with a DB is pulled down and the DB state spatially extends in the [001] (z) direction. This behavior degrades the insulating properties of the $\mathrm{Si}_{-} \mathrm{SiO}_{2}$ model. In contrast, when the DB state is inclined to be an $s$ type, the three remaining bonds tend to become $p$ types. In this case, the angular separation of these bonds is reduced from that in the tetrahedral structure, where the separation angle is $109.5^{\circ}$. As a result, the atom with the DB moves away from the three bonded atoms. Therefore, for the DB state of the raised Ge atom, the charge density of the state is distributed in the lateral directions compared with that of the Si-DB state, and the Ge-DB state is coupled with interface states of the Ge substrate [Fig. 7(b)]. This behavior barely contributes to electron transport across the model. Consequently, by introducing the oxygen vacancy, the leakage current in the $\mathrm{Si}-\mathrm{SiO}_{2}$ model increases by a factor of 162.9 , while that in the $\mathrm{Ge}-\mathrm{GeO}_{2}$ model increases by a factor of 11.8 [46].

\section{CONCLUSION}

We have presented the grid LS equation method based on the fully real-space algorithms to elucidate the scattering wave functions in nanoscale structures sandwiched between semi-infinite electrodes. It is shown that the numerical collapse due to the exponentially growing and decaying evanescent waves and the computational costs can be restrained by using the ratio expression of the retarded Green's function obtained analytically (jellium electrode case) and by incorporating the self-energy matrices and applying the recursive formulas to the ratio matrices (the crystalline electrode case). To demonstrate the performance of our method, we used it to calculate the transport properties of $(001) \mathrm{Si}-\mathrm{SiO}_{2}$ and $(001) \mathrm{Ge}-\mathrm{GeO}_{2}$ models attached to semi-infinite jellium electrodes. The results show that the $\mathrm{DB}$ state in the $\mathrm{Ge}-\mathrm{GeO}_{2}$ model gives a much smaller contribution to leakage current than that in the $\mathrm{Si}-\mathrm{SiO}_{2}$ model. Our procedure can precisely and efficiently extend the knowledge of the physics underlying the transport of electrons through nanoscale structures.

\section{ACKNOWLEDGMENTS}

This research was supported by Grant-in-Aid for Young Scientists (B) No. 24710152 from the Ministry of Education, Culture, Sports, Science and Technology. The numerical calculation was carried out using the computer facilities at the Institute for Solid State Physics at the University of Tokyo, Information Synergy Center at Tohoku University.

\section{APPENDIX A: VARIABLE-SEPARABLE-FORMED RETARDED GREEN'S FUNCTION IN THE RSFD APPROACH}

In this Appendix, the subscript 0 denoting the reference system is omitted, for simplicity. Let us consider the product of the matrix $(E-\hat{H})$ and the $l$ th columnar vector of $\hat{G}^{r}(E),\left\{G^{r}\left(\zeta_{k}, \zeta_{l} ; E\right)\right\} \quad(k=\ldots, l-1, l, l+1, \ldots)$. The retarded Green's function is constructed from outwardly propagating and decreasing waves, and then, taking it into account, we assume this columnar vector to be represented by

$$
\begin{gathered}
{\left[\ldots, U_{R}\left(\zeta_{l-2}\right) C_{R}\left(\zeta_{l}\right), U_{R}\left(\zeta_{l-1}\right) C_{R}\left(\zeta_{l}\right), D\left(\zeta_{l}\right), U_{L}\left(\zeta_{l+1}\right) C_{L}\left(\zeta_{l}\right),\right.} \\
\left.\quad U_{L}\left(\zeta_{l+2}\right) C_{L}\left(\zeta_{l}\right), \ldots\right]^{t},
\end{gathered}
$$

where $C_{R(L)}\left(\zeta_{l}\right)$ and $D\left(\zeta_{l}\right)\left[\equiv G^{r}\left(\zeta_{l}, \zeta_{l} ; E\right)\right]$ are unknown block matrices, and $U_{R(L)}\left(\zeta_{l}\right)$ is defined by Eqs. (9) and (10). Hereafter, $U_{R(L)}\left(\zeta_{l}\right), C_{R(L)}\left(\zeta_{l}\right)$, and $D\left(\zeta_{l}\right)$ are abbreviated to $U_{l}^{R(L)}, C_{l}^{R(L)}$, and $D_{l}$, respectively. By definition, the above- 
mentioned product satisfies

$$
\begin{aligned}
& \left(\begin{array}{ccccccc}
\ddots & \ddots & & & & & \\
\ddots & A_{l-2} & -B_{l-2} & & & 0 & \\
& -B_{l-2}^{\dagger} & A_{l-1} & -B_{l-1} & & & \\
& & -B_{l-1}^{\dagger} & A_{l} & -B_{l} & & \\
& & & -B_{l}^{\dagger} & A_{l+1} & -B_{l+1} & \\
& & & -B_{l+1}^{\dagger} & A_{l+2} & \ddots \\
& & & & & \ddots & \ddots
\end{array}\right) \\
& \left(\begin{array}{c}
\vdots \\
U_{l-2}^{R} C_{l}^{R} \\
U_{l-1}^{R} C_{l}^{R} \\
D_{l} \\
U_{l+1}^{L} C_{l}^{L} \\
U_{l+2}^{L} C_{l}^{L} \\
\vdots
\end{array}\right)=\left(\begin{array}{c}
\vdots \\
0 \\
0 \\
I \\
0 \\
0 \\
\vdots
\end{array}\right) \leftarrow \text { the } l \text { th }
\end{aligned}
$$

where $A_{l}=E-H\left(\zeta_{l}\right)$ and $B_{l}^{(\dagger)}=B^{(\dagger)}\left(\zeta_{l}\right)$. Since $\left\{U_{l}^{R(L)}\right\}$ is a set of the solutions of the Kohn-Sham equation, the following equations hold:

$$
\begin{gathered}
-B_{k-1}^{\dagger} U_{k-1}^{R}+A_{k} U_{k}^{R}-B_{k} U_{k+1}^{R}=0 \\
-B_{k-1}^{\dagger} U_{k-1}^{L}+A_{k} U_{k}^{L}-B_{k} U_{k+1}^{L}=0 \\
(k=\ldots, l-1, l, l+1, \ldots) .
\end{gathered}
$$

From Eqs. (A2)-(A4), one sees that the unknown matrices $C_{l}^{R(L)}$ and $D_{l}$ are required to satisfy the equations

$$
\begin{aligned}
B_{l-1} U_{l}^{R} C_{l}^{R}-B_{l-1} D_{l} & =0, \\
-B_{l-1}^{\dagger} U_{l-1}^{R} C_{l}^{R}+A_{l} D_{l}-B_{l} U_{l+1}^{L} C_{l}^{L} & =I, \\
-B_{l}^{\dagger} D_{l}+B_{l}^{\dagger} U_{l}^{L} C_{l}^{L} & =0,
\end{aligned}
$$

and thus, Eqs. (A5) and (A7) lead to the relationships between $C_{l}^{R(L)}$ and $D_{l}$ as

$$
\begin{aligned}
& C_{l}^{R}=\left(U_{l}^{R}\right)^{-1} D_{l}, \\
& C_{l}^{L}=\left(U_{l}^{L}\right)^{-1} D_{l},
\end{aligned}
$$

and Eq. (A6) determines $D_{l}$ to be

$$
D_{l}=\left[-B_{l-1}^{\dagger} U_{l-1}^{R}\left(U_{l}^{R}\right)^{-1}+A_{l}-B_{l} U_{l+1}^{L}\left(U_{l}^{L}\right)^{-1}\right]^{-1} .
$$

In consequence, the retarded Green's function $G_{T}^{r}\left(\zeta_{k}, \zeta_{l} ; E\right)$ can be described in the following separable form:

$$
G_{T}^{r}\left(\zeta_{k}, \zeta_{l} ; E\right)= \begin{cases}U_{k}^{R}\left(U_{l}^{R}\right)^{-1} D_{l} & (k<l), \\ D_{l} & (k=l), \\ U_{k}^{L}\left(U_{l}^{L}\right)^{-1} D_{l} & (k>l) .\end{cases}
$$

\section{APPENDIX B: ANALYTICAL EXPRESSION OF THE GREEN'S FUNCTION FOR A FREE ELECTRON SYSTEM IN THE RSFD APPROACH}

A one-dimensional system is first considered, for simplicity. In the RSFD approach, the kinetic-energy operator $\hat{K}=-\frac{1}{2} \nabla^{2}$ is represented by the matrix $\hat{K}^{\left(\mathcal{N}_{f}\right)}$, and the kinetic-energy term in the Kohn-Sham equation is written as

$$
\begin{aligned}
\hat{K}^{\left(\mathcal{N}_{f}\right)} \psi\left(z_{\ell}\right) \equiv & -\frac{1}{2 h^{2}}\left(C_{-\mathcal{N}_{f}} \psi\left(z_{\ell-\mathcal{N}_{f}}\right)+C_{-\mathcal{N}_{f}+1} \psi\left(z_{\ell-\mathcal{N}_{f}+1}\right)\right. \\
& +\cdots+C_{-1} \psi\left(z_{\ell-1}\right)+C_{0} \psi\left(z_{\ell}\right)+C_{1} \psi\left(z_{\ell+1}\right) \\
& \left.+\cdots+C_{\mathcal{N}_{f}-1} \psi\left(z_{\ell+\mathcal{N}_{f}-1}\right)+C_{\mathcal{N}_{f}} \psi\left(z_{\ell+\mathcal{N}_{f}}\right)\right)
\end{aligned}
$$

where $\mathcal{N}_{f}$ is the order of the finite-difference approximation, $h$ is the grid spacing, and the weight coefficients $C_{i}(i=\ell-$ $\mathcal{N}_{f}, \ell-\mathcal{N}_{f}+1, \ldots, \ell+\mathcal{N}_{f}$ ) are determined using the Taylor expansion [22].

In the $\mathcal{N}_{f}$ th-order finite-difference approximation, the Green's function matrix $\hat{G}^{\left(\mathcal{N}_{f}\right)}$ is determined as satisfying

$$
\left(Z-\hat{K}^{\left(\mathcal{N}_{f}\right)}\right) \hat{G}^{\left(\mathcal{N}_{f}\right)}(Z)=\hat{I},
$$

where $Z$ is a complex number and $\hat{I}$ is the unit matrix. The $\ell$ th row $-\ell$ 'th column element of the Green's function matrix $G^{\left(\mathcal{N}_{f}\right)}\left(z_{k}, z_{l} ; Z\right)$ is described in a spectral representation as

$$
G^{\left(\mathcal{N}_{f}\right)}\left(z_{k}, z_{l} ; Z\right)=\int_{-\pi / h}^{\pi / h} \frac{\phi_{p}\left(z_{k}\right) \phi_{p}^{*}\left(z_{l}\right)}{Z-E_{p}^{\left(\mathcal{N}_{f}\right)}} d p,
$$

where $E_{p}^{\left(\mathcal{N}_{f}\right)}$ and $\phi_{p}\left(z_{\ell}\right)$ are the eigenvalue and eigenvector of $\hat{K}^{\left(\mathcal{N}_{f}\right)}$, respectively, obtained by solving the eigenvalue equation $\hat{K}^{\left(\mathcal{N}_{f}\right)} \phi_{p}\left(z_{\ell}\right)=E_{p}^{\left(\mathcal{N}_{f}\right)} \phi_{p}\left(z_{\ell}\right)$, and are given by

$$
\begin{aligned}
E_{p}^{\left(\mathcal{N}_{f}\right)} & =-\frac{1}{2 h^{2}}\left(C_{0}+2 \sum_{m=1}^{\mathcal{N}_{f}} C_{m} \cos m p h\right), \\
\phi_{p}\left(z_{\ell}\right) & =\sqrt{\frac{h}{2 \pi}} \mathrm{e}^{i p z_{\ell}},
\end{aligned}
$$

where $-\frac{\pi}{h}<p \leqslant \frac{\pi}{h}$, and $\phi_{p}\left(z_{\ell}\right)$ is normalized, i.e.,

$$
\sum_{\ell=-\infty}^{\infty} \phi_{p}^{*}\left(z_{\ell}\right) \phi_{p^{\prime}}\left(z_{\ell}\right)=\delta\left(p-p^{\prime}\right) .
$$

Substituting Eq. (B4) into Eq. (B3) and changing the integration variable from $p$ to $\theta=p h$, and subsequently from $\theta$ to $\omega=\mathrm{e}^{i \theta}$, we obtain

$$
\begin{aligned}
& G^{\left(\mathcal{N}_{f}\right)}\left(z_{k}, z_{l} ; Z\right) \\
& =\frac{h^{2}}{2 \pi} \int_{-\pi}^{\pi} \frac{\mathrm{e}^{i \theta(k-l)}}{h^{2} Z+\frac{1}{2} C_{0}+\sum_{m=1}^{\mathcal{N}_{f}} C_{m} \cos m \theta} d \theta \\
& =\frac{h^{2}}{2 \pi i} \oint \frac{\omega^{|k-l|-1}}{h^{2} Z+\frac{1}{2} C_{0}+\frac{1}{2} \sum_{m=1}^{\mathcal{N}_{f}} C_{m}\left(\omega^{m}+\omega^{-m}\right)} d \omega .
\end{aligned}
$$


The integration can be carried out along the unit circle in the complex $\omega$ plane based on the residue theorem. In the following, we introduce a sensible manner of picking up the poles inside the unit circle that contribute to the integration. These poles $\omega$ 's are the $\mathcal{N}_{f}$ solutions of the equation

$$
h^{2} Z+\frac{1}{2} C_{0}+\frac{1}{2} \sum_{m=1}^{\mathcal{N}_{f}} C_{m}\left(\omega^{m}+\omega^{-m}\right)=0 .
$$

We now define a new variable $s$ as

$$
s=\frac{1}{2}\left(\omega+\omega^{-1}\right)=\cos \theta
$$

and rewrite Eq. (B7) as

$$
h^{2} Z+\frac{1}{2} C_{0}+\sum_{m=1}^{\mathcal{N}_{f}} C_{m} \cos m \theta=0,
$$

which is the $\mathcal{N}_{f}$ th-order algebraic equation with respect to $s$ and its solutions are denoted $s_{n}\left(n=1,2, \ldots, \mathcal{N}_{f}\right)$. Resultantly, for each $s_{n}$, the poles $\omega_{n}$ 's are given by the solutions of the quadratic equation $\omega_{n}^{2}-2 s_{n} \omega_{n}+1=0$ as

$$
\omega_{n}^{( \pm)}=s_{n} \pm \sqrt{s_{n}^{2}-1} \text {. }
$$

When the imaginary part of $Z$ is nonzero, either $\omega_{n}^{(+)}$or $\omega_{n}^{(-)}$ is inside the unit circle, while the other is outside it. Hereafter, the inside pole is defined as $\omega_{n}$, and the other is obtained by $\omega_{n}^{-1}$. Using the residue theorem, the integration of Eq. (B6) is carried out to yield

$$
\begin{aligned}
& G^{\left(\mathcal{N}_{f}\right)}\left(z_{k}, z_{l} ; Z\right) \\
& =\frac{2 h^{2}}{C_{\mathcal{N}_{f}}} \sum_{n=1}^{\mathcal{N}_{f}} \frac{\omega_{n}^{|k-l|+\mathcal{N}_{f}-1}}{\left(\omega_{n}-\omega_{n}^{-1}\right) \prod_{\substack{m=1 \\
m \neq n}}^{\mathcal{N}_{f}}\left(\omega_{n}-\omega_{m}\right)\left(\omega_{n}-\omega_{m}^{-1}\right)} \\
& =\frac{h^{2}}{2^{\mathcal{N}_{f}-2} C_{\mathcal{N}_{f}}} \sum_{n=1}^{\mathcal{N}_{f}} \frac{\omega_{n}^{|k-l|}}{\left(\omega_{n}-\omega_{m}^{-1}\right) \prod_{\substack{m=1 \\
m \neq n}}^{\mathcal{N}_{f}}\left(s_{n}-s_{m}\right)}
\end{aligned}
$$

Finally, introducing $\mathcal{K}_{n}$ by $s_{n}=\cos \mathcal{K}_{n} h$, hence, $\omega_{n}=$ $\mathrm{e}^{i \mathcal{K}_{n} h}\left(\operatorname{Im}\left\{\mathcal{K}_{n}\right\}>0\right)$, we obtain

$$
\begin{aligned}
G^{\left(\mathcal{N}_{f}\right)}\left(z_{k}, z_{l} ; Z\right)= & \frac{h^{2}}{i 2^{\mathcal{N}_{f}-1} C_{\mathcal{N}_{f}}} \\
& \times \sum_{n=1}^{\mathcal{N}_{f}} \frac{\mathrm{e}^{i \mathcal{K}_{n}\left|z_{k}-z_{l}\right|}}{\sin \mathcal{K}_{n} h \prod_{\substack{m=1 \\
m \neq n}}^{\mathcal{N}_{f}}\left(\cos \mathcal{K}_{n} h-\cos \mathcal{K}_{m} h\right)}
\end{aligned}
$$

The retarded Green's function is given by

$$
G^{\left(\mathcal{N}_{f}\right) r}\left(z_{k}, z_{l} ; E\right)=\lim _{\varepsilon \rightarrow 0^{+}} G^{\left(\mathcal{N}_{f}\right)}\left(z_{k}, z_{l} ; E+i \varepsilon\right) .
$$

It is noted that $\mathcal{K}_{n}$ is a multivalued function since it is defined as $\mathcal{K}_{n}=\frac{1}{h} \cos ^{-1} s_{n}$. The branch of $\cos ^{-1}$ should be chosen to satisfy the requirement of $\operatorname{Im}\left\{\mathcal{K}_{n}\right\}>0$, which guarantees that $\omega_{n}$ exists inside the unit circle in the complex $\omega$ plane. Thus, the following relationship is established:

$$
\begin{aligned}
& \text { If } \operatorname{Im}\left\{s_{n}\right\}<0, \text { then } 0<\operatorname{Re}\left\{\mathcal{K}_{n}\right\}<\frac{\pi}{h} . \\
& \text { If } \operatorname{Im}\left\{s_{n}\right\}>0, \text { then }-\frac{\pi}{h}<\operatorname{Re}\left\{\mathcal{K}_{n}\right\}<0 .
\end{aligned}
$$

Proof. Consider the complex numbers $s \equiv \xi+i \eta$ and $\mathcal{K} \equiv$ $k+i \kappa$ with the relationship of $s=\cos \mathcal{K} h$ in the interval of $|k|<\frac{\pi}{h}$. It is readily shown that when $\eta<0$ and $\kappa>0$, there exists a one-to-one correspondence between $\{s\}$ and $\{\mathcal{K}\}$. In this case, $\mathcal{K}$ is uniquely defined so as to satisfy $\operatorname{Im}\{\mathcal{K}\}=\kappa>0$ and the relationship

$$
\begin{aligned}
& k= \begin{cases}\frac{1}{h} \cos ^{-1} \gamma^{-} & \ldots \xi \geqslant 0, \\
\frac{1}{h} \cos ^{-1}\left(-\gamma^{-}\right) & \ldots \xi<0,\end{cases} \\
& \kappa=\frac{1}{h} \cosh ^{-1} \gamma^{+},
\end{aligned}
$$

where $\gamma^{ \pm}$is defined as

$$
\gamma^{ \pm}=\sqrt{\frac{\xi^{2}+\eta^{2}+1 \pm \sqrt{\left(\xi^{2}+\eta^{2}-1\right)^{2}+4 \eta^{2}}}{2}},
$$

and $\cos ^{-1}\left(\cosh ^{-1}\right)$ in Eq. (B15) is the principal value of the inverse trigonometric (hyperbolic) cosine function. Thus, $k$ varies as

$$
\begin{gathered}
0<k \leqslant \frac{\pi}{2 h} \ldots \xi \geqslant 0, \\
\frac{\pi}{2 h}<k<\frac{\pi}{h} \ldots \xi<0 .
\end{gathered}
$$

In the derivation of Eqs. (B15)-(B17), we used well-known formulas:

$$
\begin{aligned}
\cos (k+i \kappa) h & =\cos k h \cosh \kappa h-i \sin k h \sinh \kappa h, \\
\sin ^{2} k h+\cos ^{2} k h & =1, \\
-\sinh ^{2} \kappa h+\cosh ^{2} \kappa h & =1 .
\end{aligned}
$$

On the other hand, in the case where $\eta>0$ and $\kappa>0$, then $k$ and $\kappa$ are determined in the same manner as

$$
\begin{aligned}
& k=\left\{\begin{array}{l}
-\frac{1}{h} \cos ^{-1} \gamma^{-} \\
-\frac{1}{h} \cos ^{-1}\left(-\gamma^{-}\right) \quad \ldots \xi \geqslant 0,
\end{array}\right. \\
& \kappa=\frac{1}{h} \cosh ^{-1} \gamma^{+},
\end{aligned}
$$

respectively, and $k$ varies as

$$
\begin{aligned}
-\frac{\pi}{2 h} & \leqslant k<0 \quad \ldots \xi \geqslant 0, \\
-\frac{\pi}{h} & <k<-\frac{\pi}{2 h} \ldots \xi<0 .
\end{aligned}
$$


It is straightforward to extend the above argument to the three-dimensional case. We deal with a free electron system in which the discretized space is infinite in the $z$ direction and periodic in the $x$ and $y$ directions. The Green's function in this case is described in a spectral representation by

$$
G^{\left(\mathcal{N}_{f}\right)}\left(\boldsymbol{r}_{\|, j}, z_{k}, \boldsymbol{r}_{\|, j^{\prime}}, z_{l} ; Z\right)=\sum_{n_{x}=-\frac{N_{x}-1}{2}}^{\frac{N_{x}-1}{2}} \sum_{n_{y}=-\frac{N_{y}-1}{2}}^{\frac{N_{y}-1}{2}} \int_{-\frac{\pi}{h_{z}}}^{\frac{\pi}{h_{z}}} \frac{\phi_{n_{x}, n_{y}, p}\left(\boldsymbol{r}_{\|, j}, z_{k}\right) \phi_{n_{x}, n_{y}, p}^{*}\left(\boldsymbol{r}_{\|, j^{\prime}}, z_{l}\right)}{Z-E_{n_{x}, n_{y}, p}^{\left(\mathcal{N}_{f}\right)}} d p,
$$

where $E_{n_{x}, n_{y}, p}^{\left(\mathcal{N}_{f}\right)}$ and $\phi_{n_{x}, n_{y}, p}\left(\boldsymbol{r}_{\|, j}, z_{\ell}\right)$ are the eigenvalue and eigenvector of the three-dimensional kinetic-energy matrix, respectively; i.e.,

$$
\begin{aligned}
E_{n_{x}, n_{y}, p}^{\left(\mathcal{N}_{f}\right)} & =E_{n_{x}, n_{y}}^{\left(\mathcal{N}_{f}\right)}+E_{p}^{\left(\mathcal{N}_{f}\right)} \\
E_{n_{x}, n_{y}}^{\left(\mathcal{N}_{f}\right)} & =-\frac{1}{2 h_{x}^{2}}\left(C_{0}+2 \sum_{m=1}^{\mathcal{N}_{f}} C_{m} \cos m G_{n_{x}} h_{x}\right)-\frac{1}{2 h_{y}^{2}}\left(C_{0}+2 \sum_{m=1}^{\mathcal{N}_{f}} C_{m} \cos m G_{n_{y}} h_{y}\right), \\
E_{p}^{\left(\mathcal{N}_{f}\right)} & =-\frac{1}{2 h_{z}^{2}}\left(C_{0}+2 \sum_{m=1}^{\mathcal{N}_{f}} C_{m} \cos m p h_{z}\right) \\
\phi_{n_{x}, n_{y}, p}\left(\boldsymbol{r}_{\|, j}, z_{\ell}\right) & =\sqrt{\frac{h_{z}}{2 \pi N_{x} N_{y}}} \exp \left(i \boldsymbol{G}_{\|, n} \cdot \boldsymbol{r}_{\|, j}+i p z_{\ell}\right) .
\end{aligned}
$$

Here, $\boldsymbol{r}_{\|, j}=\left(x_{j_{x}}, y_{j_{y}}\right)$ are the lateral coordinates with $j_{x(y)}=1,2, \ldots, N_{x(y)}$ [for convenience, $N_{x(y)}$ is chosen an odd integer], and the definition of $\boldsymbol{G}_{\|, n}$ is the same as shown in Eq. (24). Now, the Green's function represented by Eq. (B12) reads as

$$
G^{\left(\mathcal{N}_{f}\right)}\left(\boldsymbol{r}_{\|, j}, z_{k}, \boldsymbol{r}_{\|, j^{\prime}}, z_{l} ; Z\right)=\frac{h_{z}^{2}}{i 2^{\mathcal{N}_{f}-1} N_{x} N_{y} C_{\mathcal{N}_{f}}} \sum_{n_{x}=-\frac{N_{x}-1}{2}}^{\frac{N_{x}-1}{2}} \sum_{n_{y}=-\frac{N_{y}-1}{2}}^{\frac{N_{y}-1}{2}} \sum_{n=1}^{\mathcal{N}_{f}} \frac{\exp \left[i \boldsymbol{G}_{\|, n} \cdot\left(\boldsymbol{r}_{\|, j}-\boldsymbol{r}_{\|, j^{\prime}}\right)+i \mathcal{K}_{n}\left|z_{k}-z_{l}\right|\right]}{\sin \mathcal{K}_{n} h_{z} \prod_{\substack{m=1 \\ m \neq n}}^{\mathcal{N}_{f}}\left(\cos \mathcal{K}_{n} h_{z}-\cos \mathcal{K}_{m} h_{z}\right)}
$$

where $\mathcal{K}_{n}=\frac{1}{h_{z}} \cos ^{-1} s_{n}$ under the requirement of $\operatorname{Im}\left\{\mathcal{K}_{n}\right\}>0$ and $s_{n}$ is the solution of the $\mathcal{N}_{f}$ th-order algebraic equation with respect to $s(=\cos \theta)$,

$$
h_{z}^{2}\left(Z-E_{n_{x}, n_{y}}^{\left(\mathcal{N}_{f}\right)}\right)+\frac{1}{2} C_{0}+\sum_{m=1}^{\mathcal{N}_{f}} C_{m} \cos m \theta=0 .
$$

In the following, we present the analytic representation of the retarded Green's functions in $\mathcal{N}_{f}=1-4$ cases in a one-dimensional free electron system; there exists no analytic representation in the case of $\mathcal{N}_{f} \geqslant 5$, since the algebraic equation, (B9), with $\mathcal{N}_{f} \geqslant 5$ cannot be solvable analytically according to Galois theory. Given the solutions of Eq. (B9), $s_{n} \equiv \xi_{n}+i \eta_{n}\left(n=1,2, \ldots, \mathcal{N}_{f}\right), K_{n} \equiv k_{n}+i \kappa_{n}$ are determined from Eqs. (B14)-(B20), and finally, we obtain the analytic representation of the Green's function, Eq. (B12). Hereafter, we choose $Z=E+i \varepsilon$ ( $\varepsilon$ : an infinitesimal positive number) in Eq. (B12) so as to deal with the retarded Green's function.

\section{Case of a central finite difference $\left(\mathcal{N}_{f}=1\right)$}

Substituting $C_{0}=-2$ and $C_{1}=1$ into Eq. (B9), we have the equation

$$
s-1+h^{2}(E+i \varepsilon)=0
$$

and its solution

$$
s_{1} \equiv \xi_{1}+i \eta_{1}=1-h^{2} E-i h^{2} \varepsilon
$$

Since Eq. (B26) indicates that $\eta_{1} \rightarrow 0^{-}$(an infinitesimal negative number) in the limit of $\varepsilon \rightarrow 0^{+}, \mathcal{K}_{1} \equiv k_{1}+i \kappa$ is determined from Eqs. (B15) and (B16) such that

$$
\begin{array}{rlrl}
k_{1} & =\frac{1}{h} \cos ^{-1}\left(1-h^{2} E\right), & \kappa_{1} & =0 \\
k_{1} & =0, & \ldots 0 \leqslant E<\frac{2}{h^{2}} \\
k_{1} & =\frac{1}{h} \cosh ^{-1}\left(1-h^{2} E\right) \ldots E<0 ; \\
k_{1} & =\frac{\pi}{h}, & \kappa_{1} & =\frac{1}{h} \cosh ^{-1}\left(-1+h^{2} E\right) \ldots \frac{2}{h^{2}} \leqslant E .
\end{array}
$$


2. Case of a five-point finite difference $\left(\mathcal{N}_{f}=2\right)$

Substituting $C_{0}=-5 / 2, C_{1}=4 / 3$, and $C_{2}=-1 / 12$ into Eq. (B9) yields the quadratic equation with respect to $s$,

$$
s^{2}-8 s+7-6 h^{2}(E+i \varepsilon)=0 .
$$

This equation has two solutions, $s_{1}$ and $s_{2}$, given by

$s_{1} \equiv \xi_{1}+i \eta_{1}=4-\sqrt{9+6 h^{2}(E+i \varepsilon)}, \quad$ where $\begin{cases}\xi_{1}=4-\sqrt{9+6 h^{2} E}, & \eta_{1} \rightarrow 0^{-} \\ \xi_{1}=4, & \eta_{1}=-\sqrt{\left|9+6 h^{2} E\right|<0 \ldots E<-\frac{3}{2 h^{2}},},\end{cases}$

and

$s_{2} \equiv \xi_{2}+i \eta_{2}=4+\sqrt{9+6 h^{2}(E+i \varepsilon)}, \quad$ where $\left\{\begin{array}{ll}\xi_{2}=4+\sqrt{9+6 h^{2} E}, & \eta_{2} \rightarrow 0^{+} \\ \xi_{2}=4, & \eta_{2}=\sqrt{\left|9+6 h^{2} E\right|>0} \ldots E<-\frac{3}{2 h^{2}} \leqslant E,\end{array} \quad\right.$.

Subsequently, from Eqs. (B15), (B16), and (B19), $\mathcal{K}_{n} \equiv k_{n}+i \kappa_{n}$ can be described in an analytic form.

3. Case of a seven-point finite difference $\left(\mathcal{N}_{f}=3\right)$

The substitution of $C_{0}=-49 / 18, C_{1}=3 / 2, C_{2}=-3 / 20$, and $C_{3}=1 / 90$ into Eq. (B9) leads to the cubic equation with respect to $s$,

$$
s^{3}-\frac{27}{4} s^{2}+33 s-\frac{109}{4}+\frac{45}{2} h^{2}(E+i \varepsilon)=0,
$$

whose solutions are determined according to Cardano's formula as

$$
\begin{array}{lll}
s_{1} \equiv \xi_{1}+i \eta_{1}, \quad \text { where } \quad \xi_{1}=\frac{9}{4}-\tau^{+}+\tau^{-} & \text {and } \quad \eta_{1} \rightarrow 0^{-}, \\
s_{2} \equiv \xi_{2}+i \eta_{2}, & \text { where } \quad \xi_{2}=\frac{\tau^{+}-\tau^{-}}{2} \quad \text { and } \quad \eta_{2}=\frac{\tau^{+}+\tau^{-}}{2}>0, \\
s_{3} \equiv \xi_{3}+i \eta_{3}, & \text { where } \quad \xi_{3}=\frac{\tau^{+}-\tau^{-}}{2} \quad \text { and } \quad \eta_{3}=-\frac{\tau^{+}+\tau^{-}}{2}<0 .
\end{array}
$$

Here,

$$
\tau^{ \pm}=\frac{1}{4} \sqrt[3]{5\left[\sqrt{\left(144 h^{2} E+155\right)^{2}+5 \times 19^{3}} \pm\left(144 h^{2} E+155\right)\right]},
$$

and $\mathcal{K}_{n} \equiv k_{n}+i \kappa_{n}$ can be analytically represented using Eqs. (B15), (B16), and (B19).

\section{Case of a nine-point finite difference $\left(\mathcal{N}_{f}=4\right)$}

Now, substituting $C_{0}=-205 / 72, C_{1}=8 / 5, C_{2}=-1 / 5, C_{3}=8 / 315$, and $C_{4}=-1 / 560$ into Eq. (B9), we obtain the quartic equation with respect to $s$,

$$
s^{4}-\frac{64}{9} s^{3}+27 s^{2}-\frac{320}{3} s+\frac{772}{9}-70 h^{2}(E+i \varepsilon)=0 .
$$

Ferrari's solutions to Eq. (B36) are utilized. After tedious but straightforward calculations, we have the following representations:

$$
\begin{aligned}
& s_{1} \equiv \xi_{1}+i \eta_{1}, \quad \text { where }\left\{\begin{array}{lll}
\xi_{1}=\frac{16}{9}+\sigma-\sqrt{-\sigma^{2}-\alpha+\frac{\beta}{\sigma}}, & \eta_{1} \rightarrow 0^{-} & \ldots E_{0} \leqslant E, \\
\xi_{1}=\frac{16}{9}+\sigma, & \eta_{1}=-\sqrt{\sigma^{2}+\alpha-\frac{\beta}{\sigma}}<0 & \cdots E<E_{0} ;
\end{array}\right. \\
& s_{2} \equiv \xi_{2}+i \eta_{2}, \quad \text { where }\left\{\begin{array}{lll}
\xi_{2}=\frac{16}{9}+\sigma+\sqrt{-\sigma^{2}-\alpha+\frac{\beta}{\sigma}}, & \eta_{2} \rightarrow 0^{+} & \ldots E_{0} \leqslant E, \\
\xi_{2}=\frac{16}{9}+\sigma, & \eta_{2}=\sqrt{\sigma^{2}+\alpha-\frac{\beta}{\sigma}}>0 & \cdots E<E_{0} ;
\end{array}\right.
\end{aligned}
$$




$$
\begin{aligned}
& s_{3} \equiv \xi_{3}+i \eta_{3}, \quad \text { where }\left\{\begin{array}{lll}
\xi_{3}=\frac{16}{9}-\sigma, & \eta_{3}=-\sqrt{\sigma^{2}+\alpha+\frac{\beta}{\sigma}}<0 \quad \ldots E_{0}^{\prime} \leqslant E, \\
\xi_{3}=\frac{16}{9}-\sigma, & \eta_{3}=\sqrt{\sigma^{2}+\alpha+\frac{\beta}{\sigma}}>0 \quad \ldots E<E_{0}^{\prime} ;
\end{array}\right. \\
& s_{4} \equiv \xi_{4}+i \eta_{4}, \quad \text { where }\left\{\begin{array}{lll}
\xi_{4}=\frac{16}{9}-\sigma, & \eta_{4}=\sqrt{\sigma^{2}+\alpha+\frac{\beta}{\sigma}}>0 \quad \ldots E_{0}^{\prime} \leqslant E, \\
\xi_{4}=\frac{16}{9}-\sigma, & \eta_{4}=-\sqrt{\sigma^{2}+\alpha+\frac{\beta}{\sigma}}<0 \quad \ldots E<E_{0}^{\prime} .
\end{array}\right.
\end{aligned}
$$

Here,

$$
\begin{aligned}
\alpha & =\frac{7 \cdot 31}{2 \cdot 3^{3}}, \quad \beta=\frac{2^{3} \cdot 7 \cdot 181}{3^{6}}, \\
\sigma & =\left\{\begin{array}{l}
\sqrt{\alpha\left(-\frac{1}{3}+\sqrt[3]{\rho+\sqrt{v^{3}+\rho^{2}}}-\sqrt[3]{-\rho+\sqrt{v^{3}+\rho^{2}}}\right)} \ldots 0 \leqslant v, \\
\sqrt{\alpha\left(-\frac{1}{3}+\sqrt[3]{\rho+\sqrt{v^{3}+\rho^{2}}}+\sqrt[3]{\rho-\sqrt{v^{3}+\rho^{2}}}\right)} \ldots v<0,
\end{array}\right. \\
\nu & =a_{0}+a_{1} h^{2} E, \quad \rho=\frac{1}{2} v+a_{2}, \\
E_{0} & =\frac{v_{0}-a_{0}}{a_{1} h^{2}}, \quad E_{0}^{\prime}=\frac{v_{0}^{\prime}-a_{0}}{a_{1} h^{2}}, \\
a_{0} & =\frac{3^{2} \cdot 5 \cdot 19}{2^{2} \cdot 31^{2}}, \quad a_{1}=\frac{2 \cdot 3^{5} \cdot 5}{7 \cdot 31^{2}}, \quad a_{2}=\frac{3^{2} \cdot 5 \cdot 3623}{2 \cdot 7 \cdot 31^{3}},
\end{aligned}
$$

and $v_{0}$ is the solution of $v^{3}+\rho^{2}=0$, which is evaluated as $v_{0} \approx-0.3563$, and $v_{0}^{\prime}=-\left(\left(a_{3}+1 / 3\right)^{3}-2 a_{2}\right) / 3 a_{3} \approx-0.4132$, with $a_{3}$ being $\sqrt[3]{2 \beta^{2}} / 2 \alpha$. The use of Eqs. (B12)-(B20) leads to the analytic representations of $\mathcal{K}_{n} \equiv k_{n}+i \kappa_{n}$ and the retarded Green's function.

The above treatment for analytically describing the retarded Green's function is readily extendable to the three-dimensional case using Eqs. (B22)-(B24).

[1] N. D. Lang and A. R. Williams, Phys. Rev. B 18, 616 (1978).

[2] N. D. Lang, Phys. Rev. B 52, 5335 (1995).

[3] N. D. Lang and P. Avouris, Phys. Rev. B 64, 125323 (2001).

[4] N. Kobayashi, M. Aono, and M. Tsukada, Phys. Rev. B 64, 121402 (2001).

[5] S. Tsukamoto and K. Hirose, Phys. Rev. B 66, 161402(R) (2002).

[6] S. Tsukamoto, M. Aono, and K. Hirose, Jpn. J. Appl. Phys. 41, 7491 (2002).

[7] J. Nara, W. T. Geng, H. Kino, N. Kobayashi, and T. Ohno, J. Chem. Phys. 121, 6485 (2004).

[8] S. Tsukamoto, Y. Egami, K. Hirose, and S. Blügel, Phys. Rev. B 84, 115443 (2011).

[9] Y. Fujimoto and K. Hirose, Phys. Rev. B 67, 195315 (2003).

[10] K. Hirose, T. Ono, Y. Fujimoto, and S. Tsukamoto, FirstPrinciples Calculations in Real-Space Formalism, Electronic Configurations and Transport Properties of Nanostructures (Imperial College Press, London, 2005).

[11] T. Ono and K. Hirose, Phys. Rev. Lett. 82, 5016 (1999).

[12] K. Hirose and T. Ono, Phys. Rev. B 64, 085105 (2001).

[13] T. Ono and K. Hirose, Phys. Rev. B 72, 085105 (2005).

[14] T. Ono and K. Hirose, Phys. Rev. B 72, 085115 (2005).
[15] T. Sasaki, T. Ono, and K. Hirose, Phys. Rev. E 74, 056704 (2006).

[16] T. Ono, M. Heide, N. Atodiresei, P. Baumeister, S. Tsukamoto, and S. Blügel, Phys. Rev. B 82, 205115 (2010).

[17] Y. Egami, K. Hirose, and T. Ono, Phys. Rev. E 82, 056706 (2010).

[18] T. Ono, Y. Egami, and K. Hirose, Phys. Rev. B 86, 195406 (2012).

[19] P. Hohenberg and W. Kohn, Phys. Rev. 136, B864 (1964).

[20] W. Kohn and L. J. Sham, Phys. Rev. 140, A1133 (1965).

[21] Y. Hasegawa, J.-I. Iwata, M. Tsuji, D. Takahashi, A. Oshiyama, K. Minami, T. Boku, F. Shoji, A. Uno, M. Kurokawa, H. Inoue, I. Miyoshi, and M. Yokokawa, in Proceedings of 2011 International Conference for High Performance Computing, Networking, Storage and Analysis, SC'11 (ACM, New York, 2011), p. 1; available at: http://sc11.supercomputing.org/?pg=awards.html; http://awards.acm.org/award_winners/hasegawa_7239126.cfm.

[22] J. R. Chelikowsky, N. Troullier, K. Wu, and Y. Saad, Phys. Rev. B 50, 11355 (1994).

[23] J. R. Chelikowsky, N. Troullier, and Y. Saad, Phys. Rev. Lett. 72, 1240 (1994).

[24] N. D. Lang, Phys. Rev. Lett. 79, 1357 (1997). 
[25] N. Kobayashi, M. Brandbyge, and M. Tsukada, Surf. Sci. 433435, 854 (1999); Phys. Rev. B 62, 8430 (2000).

[26] M. Okamoto, T. Uda, and K. Takayanagi, Phys. Rev. B 64, 033303 (2001).

[27] P. Havu, T. Torsti, M. J. Puska, and R. M. Nieminen, Phys. Rev. B 66, 075401 (2002).

[28] S. Furuya, Y. Gohda, and S. Watanabe, Jpn. J. Appl. Phys. 45, 8991 (2006).

[29] M. Otani, T. Ono, and K. Hirose, Phys. Rev. B 69, 121408(R) (2004); T. Ono and K. Hirose, Phys. Rev. Lett. 94, 206806 (2005); 98, 026804 (2007); Y. Egami, S. Aiba, K. Hirose, and T. Ono, J. Phys.: Cond. Matt. 19, 365201 (2007).

[30] T. Ono, J. Phys. Chem. C 113, 6256 (2009).

[31] An alternative derivation of Eq. (32) is given in Ref. [17], in which the retarded self-energy matrix of the jellium electrode, $\Sigma^{r}\left(z_{k}\right)$, is analytically calculated. See Eq. (38) for the relationship between $X_{k}$ and $\Sigma^{r}\left(z_{k}\right)$, and note also that $B\left(z_{k}\right)=-\frac{1}{2 h_{2}^{2}} I$ in the central finite-difference $\left(\mathcal{N}_{f}=1\right)$ case.

[32] H. Kageshima and K. Shiraishi, Phys. Rev. Lett. 81, 5936 (1998).

[33] M. Houssa, G. Pourtois, M. Caymax, M. Meuris, M. M. Heyns, V. V. Afanas'ev, and A. Stesmans, Appl. Phys. Lett. 93, 161909 (2008).
[34] M. Yang, R. Q. Wu, Q. Chen, W. S. Deng, Y. P. Feng, J. W. Chai, J. S. Pan, and S. J. Wang, Appl. Phys. Lett. 94, 142903 (2009).

[35] J. F. Binder, P. Broqvist, and A. Pasquarello, Microelectron. Eng. 86, 1760 (2009).

[36] P. Broqvist, J. F. Binder, and A. Pasquarello, Appl. Phys. Lett. 94, 141911 (2009).

[37] M. Houssa, E. A. Chagarov, and A. C. Kummel, MRS Bull. 34, 504 (2009).

[38] L. Tsetseris and S. T. Pantelides, Appl. Phys. Lett. 95, 262107 (2009).

[39] T. Ono, Phys. Rev. B 79, 195326 (2009).

[40] S. Saito and T. Ono, Jpn. J. Appl. Phys. 50, 021503 (2011).

[41] S. Saito and T. Ono, Phys. Rev. B 84, 085319 (2011).

[42] T. Ono and S. Saito, Appl. Phys. Exp. 4, 021303 (2011).

[43] J. P. Perdew and A. Zunger, Phys. Rev. B 23, 5048 (1981).

[44] M. Büttiker, Y. Imry, R. Landauer, and S. Pinhas, Phys. Rev. B 31, 6207 (1985).

[45] D. Haneman, Phys. Rev. 121, 1093 (1961).

[46] In each model, the leakage current was calculated by integrating the tunneling current over the energy ranges between the VBM and the CBM of the substrate. 